\title{
Insight into SNPs and epitopes of E protein of newly emerged genotype-I isolates of JEV from Midnapur, West Bengal, India
}

\author{
Shyamashree Banerjee, Parth Sarthi Sen Gupta and Amal Kumar Bandyopadhyay*
}

\begin{abstract}
Background: Japanese encephalitis virus (JEV) is a mosquito-borne flavivirus that causes Japanese Encephalitis (JE) and Acute Encephalitis Syndrome (AES) in humans. Genotype-I (as co-circulating cases with Genotype-III) was isolated in 2010 (JEV28, JEV21) and then in 2011 (JEV45) from Midnapur district, West Bengal (WB) for the first time from clinical patients who were previously been vaccinated with live attenuated SA14-14-2 strain. We apply bioinformatics and immunoinformatics on sequence and structure of E protein for analysis of crucial substitutions that might cause the genotypic transition, affecting protein-function and altering specificity of epitopes.

Results: Although frequency of substitutions in E glycoprotein of JEV28, JEV21 and JEV45 isolates vary, its homologous patterns remain exactly similar as earlier Japan isolate (Ishikawa). Sequence and 3D model-structure based analyses of $\mathrm{E}$ protein show that only four of all substitutions are critical for genotype-I specific effect of which N103K is common among all isolates indicating its role in the transition of genotype-III to genotype-I. Predicted B-cell and T-cell epitopes are seen to harbor these critical substitutions that affect overall conformational stability of the protein. These epitopes were subjected to conservation analyses using a large set of the protein from Asian continent.
\end{abstract}

Conclusions: The study identifies crucial substitutions that contribute to the emergence of genotype-I. Predicted epitopes harboring these substitutions may alter specificity which might be the reason of reported failure of vaccine. Conservation analysis of these epitopes would be useful for design of genotype-l specific vaccine.

Keywords: Japanese encephalitis virus, Genotype I, Genotype III, Midnapur, Homology model, SNP Energetics, B-cell \& T-cell epitopes, PEP-FOLD, Docking

\section{Background}

Japanese encephalitis virus (JEV), a mosquito-borne flavivirus of the family Flaviviridae, is the sole etiologic agent of Japanese encephalitis (JE). JE is the neurotropic disease has been the primary health concern in human worldwide mostly affecting children and older person. Approximately $25-30 \%$ of JEV cases are fatal and 50\% result in permanent neuropsychiatric sequelae $[1,2]$.

In India, JE was first reported in Vellore in 1955 [1, 2]. Since then, epidemics of JE in different states have been reported including large and severe outbreak in the State of Uttar Pradesh (UP) and part of Bihar that caused 5737 JE cases and 1,334 deaths in UP and 360 cases with 64 deaths

\footnotetext{
* Correspondence: akbanerjee@biotech.buruniv.ac.in Department of Biotechnology, The University of Burdwan, Burdwan, West Bengal 713104, India
}

(c) The Author(s). 2017 Open Access This article is distributed under the terms of the Creative Commons Attribution 4.0 International License (http://creativecommons.org/licenses/by/4.0/), which permits unrestricted use, distribution, and reproduction in any medium, provided you give appropriate credit to the original author(s) and the source, provide a link to the Creative Commons license, and indicate if changes were made. The Creative Commons Public Domain Dedication waiver (http://creativecommons.org/publicdomain/zero/1.0/) applies to the data made available in this article, unless otherwise stated. in Bihar in 2005 [1]. Analyses showed that genotype III (GIII) is predominant in India. However, genotype I (GI) has recently been introduced in different States in India including West Bengal [2]. Since 1973, JE outbreaks have been recorded in different districts of West Bengal (WB). Although, The State Health Department, Govt. of WB routinely conducts vaccination program (live attenuated SA1414-2, GIII) against JE cases in different districts of WB, sporadic JE cases and deaths are being reported every year. The vaccination program is challenged by the repeated observation of co-circulation cases of GIII and GI in prevaccinated patients in the district of Midnapur of WB [3, 4].

Viral genome produces single polyprotein which is processed by viral and host proteases into structural (capsid C, precursor membrane prM and envelope $\mathrm{E}$ ) and nonstructural (NS1, NS2A, NS2B, NS3, NS4A, NS4B and NS5) proteins $[5,6]$. Although all these proteins are important 
for viral life cycle, the E protein is responsible for host cell interaction, infectivity and entry of the virus [6]. Amino acid sequence of full length genome of virulent wild-type (WT) JEV strain (SA14) when compared with that of nonvirulent live-attenuated SA14-14-2 vaccine strain, it was found that a multitude of mutations are accumulated throughout the genome of the later. These genome wide mutations are collectively considered to be responsible for non-virulent property of SA14-14-2. E protein accumulates maximum number of mutations in the attenuation process and thus it is considered as the primary genetic determinant for neurovirulence and neuroinvasiveness of WT strains of JEV [5, 7]. However, detailed mechanism for neurovirulence and neuroinvasiveness in relation to locus specific amino acid changes is unknown.

E protein based phylogenetic analyses have established five different genotypes of JEV of which GIII constitutes the major genotype in Asian countries including China, Japan, Taiwan, India, Korea, Thailand, Indonesia, and Vietnam. Genotyping is commonly achieved by construction of phylogenetic tree using E protein of isolates and obtaining clades [8]. Although in low frequency, GI is seen to replace the existing GIII from all these countries [9]. In India like other countries, GIII is the dominant genotype for all states. However, co-circulation of GI with GIII in AES/JE patients was first documented from Gorakhpur/UP/India region in 2009 [9] and then from Midnapur district of West Bengal (WB), India in 2010 and 2011 [3, 4]. In the later case emergence of GI with GIII was observed in patient even when it was reported to be immunized with SA14-14-2 vaccine prior infection $[4,10]$. Similar observation is also available from China [11].

In general the sequence of $\mathrm{E}$ protein of virulent isolate of JEV is compared with that of non-virulent SA14-14-2 to gain insight into neurovirulence and neuroinvasiveness effects of the former. While high neurovirulence of WT JEV isolates was reported to be contributed by eight mutations (F107L, K138E, V176I, A177T, H264Q, M279K, V315A and $\mathbf{R 4 3 9 K )}$ in E protein, neuroinvasiveness involves mutations in other proteins also [12-15]. Notably both GI (JEV45) and GIII (JEV46 and JEV47) isolates of WB were demonstrated to be neurovirulent and possess the above mutations in $\mathrm{E}$ protein [4]. Additional substitutions observed in these isolates were claimed to have potential for i] altering immunogenicity [based on HLA (Human leukocyte antigen) class I specific decreased/increased binding scores] and ii] escape of antibody neutralization based on their occurrence in known loop structures of $\mathrm{E}$ protein of West Nile Virus and decreased/increased hydrophilicity values [4]. However, the question that which among all mutations may have relation with the observed genotypic transition, alteration of epitope's specificity and E protein function remains to be worked out. Reported failure of conventional vaccination and emergence of GI in the region needs careful prediction of B-cell and T-cell epitopes to improve immunization in the locality at high risk of disease.

In the present study we consider $\mathrm{E}$ protein of newly emerged GI isolates from Midnapur/WB to develop homology model structures possessing either all or single mutation. We then use both mutant structure and sequence based methods to identify critical among all acquired substitutions. Linear as well as conformational B-cell and Tcell (HLA class I and II specific) epitopes are also been worked out. We further report improved filtering of epitopes using conservation, accessibility, conformational stability and docking criteria. Using large database of $E$ protein from Asian countries, genotypic diversity of the epitopes is established in this study. Taken together, the study identifies GI specific critical SNPs and epitopes and finds application in immunoinformatics.

\section{Methods}

Protein sequence retrieval and analysis

Full length nucleotide sequences of $\mathrm{E}$ protein that are isolated from Midnapur district, WB, India as GI were retrieved from GenBank database [ID: JN703381 (JEV28/ 2010); JN703382 (JEV21/2010); KC526872 (JEV45/2011)]. We also procured Ishikawa/Japan GI [ID: AB051292] for comparison. The nucleotide sequences were converted into proteins using EMBOSS (European Molecular Biology Open Software Suite) Transeq tool [16]. Primary structure analysis was performed using our laboratory procedures PHYSICO and PHYSICO2 $[17,18]$. Secondary structure analysis was performed using SOPMA [19].

\section{Homology modeling, energy minimization, evaluation and submission}

Homology modeling, energy minimization and evaluation have been routine procedures for many proteins [20-23]. Crystal structure of JEV ecto domain of E protein of SA1414-2 (GIII) is available in the Protein Data Bank (PDB) with ID 3P54.pdb (Chain A, Resolution 2.1 $)$ ). The PDB sequence (UNIPROT ID: P27395) has 97.3, 96.3, 96.3 and 95.3\% identity with that of JEV28 (JN703381), JEV21 (JN703382), JEV45 (KC526872) and Ishikawa (AB051292) isolates respectively. There is no INDEL region for any of these sequences when aligned with reference template sequence (P27395). We therefore performed homology modeling on the target sequences using 3P54_A as template. Comparative model of the target sequences were achieved by MODELLER 9 v11 package [24] that makes use of spatial restraints and other optimization procedures. Alignments between template and target sequences were performed by using MODELLER in-built script. Manual improvements were incorporated in the alignment when it was necessary. At least five replicates of model were produced of which the best model was selected based on Discrete Optimized Protein Energy (DOPE) [25] assessment 
scores. Idealization of bond geometry, optimization of loop regions and removal of unwanted non-bonded contacts of the initial model was achieved by energy minimization using CHARMM (Chemistry at HARvard Macromolecular Mechanics) force field of NAMD (Nanoscale Molecular Dynamics) package [26]. To retain known disulfide bond specificity, protein's residue pairs are appropriately patched. Explicit water box of $5 \AA$ additional distance from the highest coordinate in each dimension with an exclusion radius 2.4 $\AA$ was incorporated with the model using VMD (Visual molecular dynamics) in-built solvate v1.5 plugin [27] and subjected for minimization. After 5000 steps of conjugate gradient minimization, trajectory analyses were performed by using Vega ZZ interface [28] to select the lowest energy frame of models. The resulted models were then subjected for a series of tests such as stereo-chemical analyses by PROCHECK [29] geometric relationship among nonbonded atoms by ERRAT [30], proper threading of target sequence with the model structure by VERIFY3D [31] and structural features based overall quality by ProQ. [32]. Ionpair specificity and energetics of models and template were compared using SBION [33], SBION2 [34] and ADSBET2 [35]. High quality models thus obtained were deposited in the Protein Model Data Base (PMDB) [36] and the PMDB identifiers were assigned as PM0080323, PM0079295, PM0080324 and PM0080325 for JEV28, JEV21, JEV45 and Ishikawa respectively.

We also developed model for each positional mutation observed in GI's E protein. In this procedure, we first generated target sequence from template (3P54_A) by giving one observed mutation at a time. The derived target sequence was then used for model development as described. Each model was evaluated as mentioned above. These mutant structures were used along with the template (WT) to evaluate the effect of mutation (see below).

\section{Disease related SNP prediction}

The single nucleotide polymorphism occurring in the protein coding region may lead to deleterious consequences and might affect epitopes conformational properties in protein. In order to identify disease-associated SNPs, we used SIFT [37], PhD-SNP [38] SNAP [39] and METASNP [40] authentic web server for sequence based and SDM (Site Directed Mutator) [41] web server for structure based prediction. SIFT prediction is based on the sequence homology and the physicochemical properties of amino acids which are dictated by the substituted amino acid. PhD-SNP uses support vector machines method based human deleterious SNP. It predicts whether the given amino acid substitution leads to disease associated or neutral. META-SNP is a high accuracy integrated method to discriminate between disease-related and polymorphic non-synonymous single nucleotide variants. SDM is a statistical method for calculation of difference in free energy between wild type and mutant protein and prediction of disease association.

\section{B-cell and T-cell epitope prediction}

Prediction of both $\mathrm{B}$ cell and $\mathrm{T}$ cell epitopes is the first step for rational vaccine design. Sequences of $E$ protein of GI isolates (Ishikawa, JEV28, JEV21 and JEV45) were used for prediction of B-cell linear epitopes using BCPREDS v1.0 [42] web server. Epitopes thus obtained were subjected for further screening based on various criteria such as i] model energy [43], ii] average side chain accessibility using NACCESS procedure [44], iii] Shannon Entropy based conservation properties [17, 18] and iv] chances to harbor critical substitutions of epitopes. Finally only 8 epitopes were selected.

Conformational B cell epitopes are determined using high accuracy web server EPSVR [45] using model 3D structures as input. The method uses support vector regression for prediction. The result file represents residues in color codes from low (blue i.e. $\leq 40$ ) to high (red i.e. $\geq 90$ ). Only the high possibility residues $(\geq 80 \%)$ are highlighted in the model structure using VMD interface [27].

Cellular peptide vaccine contains T-cell epitopes that bind structurally to highly diverse, polygenic and polymorphic human MHCs (Major histocompatibility complex) whose experimental determination are cost effective and time consuming. IEDB analysis tools [46] were used for MHC class specific (class I and II) T cell epitopes prediction. All available MHC class specific HLA-alleles were tested for their efficacy in binding epitopes under the strict cutoff of percentile rank (equivalent to $\mathrm{IC}_{50}$ value). Clustering of alleles against each top score epitope was performed in excel. Epitopes were further short listed by their antigenicity scores using VaxiJen v2.0 server [47]. MHC class I epitopes were further screened based on proteosomal processing score. Each set of epitopes were further short listed using average conservation $[17,18]$ and accessibility [44] value. Finally only best 10 epitopes from each class (MHC - I and MHC - II) were retained along with their respective HLA alleles.

\section{Structure prediction of epitopes and docking study}

Docking of T-cell class specific epitope on known binding site (PBG: peptide binding groove) of respective MHC class specific PDB structure was performed for further short listing. Optimized epitope structure was obtained using HMM-SA (Hidden Markov Model-derived Structural Alphabet) [48] with OPEP (Optimized Potential for Efficient protein structure Prediction) force field [49]. $2 \mathrm{X} 4 \mathrm{O}$ and 1DLH were used as peptide targets for MHC class I and II respectively. Both these targets were in complex form with inhibitors. Structure with single binding site and free from inhibitor was procured using VMD interface [27]. Binding sites of each target was mapped in 
presence of inhibitor using NACCESS procedure [44]. Docking of above epitopes (10 from each class) onto its respective targets (2X4O and $1 \mathrm{DLH})$ were performed using PatchDock server [50] along with input of computed binding site. Epitope-receptor complex was then refined using FireDock [51] server. Best four epitopes from each class were selected on the basis of energetics and interaction criteria.

\section{Results}

Like other countries in Asia [2, 8], JEV mediated acute encephalitis syndrome and associated damage and death in patients are the major health concern in India in different states including WB $[3,4,9]$. The report of cocirculation of GI with GIII in the district of Midnapur of WB increases the risk of possible outbreak in the locality. The appearance of the former was documented even when Government aided regular vaccination program was in vogue [3]. Such threatening genotypic transition and failure of neutralization raise the possibility of critical mutations in envelope glycoprotein. In this study we therefore investigate substitutions in $\mathrm{E}$ protein of GI strains (Ishikawa, JEV28, JEV21 and JEV45) in reference to the GIII vaccine strain (SA14-14-2).

\section{E protein of Gl strain acquires domain specific substitutions}

Which substitutions contribute to the transition from existing GIII to emerging GI? To check this we have presented observed substitutions in $\mathrm{E}$ protein of GI isolates in Table 1 along with reference GIII strains. Ishikawa, a typical GI isolate that caused outbreak in Japan in 1994 and possesses similar mutations at homologous positions as WB isolates is also included for comparison purpose. Six mutations: K138E, V176I, A177T in Domain I and F107L, H264Q, M279K in domain II of E protein (Table 1, marked with ${ }^{\text {a }}$ sign) are common in all the GI isolates. They are also present in virulent GIII strain SA14 (Table 1: column 8) from which the live attenuated non-virulent SA14-14-2 strain was derived. Notably, north India epidemic (in 2005) strain GP78 (ID AF075723) [1] and recent GIII isolates (JEV46 and JEV47) from WB [4] also contain the above six mutations. As these mutations in E protein are common in SA14, GI and GIII virulent isolates, net mutations in our GI isolates are counted without them. However following points are noteworthy from the Table 1: i] WB isolates have similar positional substitutions as earlier Japan isolate (Ishikawa) which has highest number of mutations in all three domains of E protein, ii] JEV28 (2010) has fewer mutations in most immunogenic domain III than JEV21 (2010) and JEV45 (2011). Substitutions V372L, M374I, G388K, W396R are absent in JEV28, iii] four amino acid changes in PDB (3P54_A) sequence relative to
UNIPROT one (ID P27395): K138E, V176I, F107L and M279K were considered as mismatch [52].

To check domain distribution of net substitutions (Table 1) of E protein, we have presented the following Figure (Fig. 1). Several points are noteworthy from the Figure. Firstly as we move from DI to DIII, substitution frequency increases both for Ishikawa and JEV45 isolates. The later, just one year younger than JEV28/2010, is seen to acquire almost identical loci specific substitutions as Ishikawa/Japan/1994. Secondly, as far as total substitutions are concerned, Ishikawa dominates over both JEV28 and JEV45. Finally, relative to JEV28 three additional substitutions in JEV45 are seen in domain-III. The domain is reported to be most immunogenic [53].

\section{Structural insight into $\mathrm{E}$ proteins of $\mathrm{Gl}$ isolates}

The ecto domain of $E$ protein is composed of 406 residues that contains majority of the antigenic determinants. The sequence identity of E protein of Ishikawa, JEV28 and JEV45 are 94.8, 96.8 and $95.8 \%$ respectively relative to SA14-14-2. Primary and secondary structural properties of E protein of these GI isolates (Ishikawa, JEV28 and JEV45) are compared with reference sequence (SA14-142) in Fig. 2. It is seen that most of physicochemical properties, except GRAVY (Grand Average Hydropathy), net charge and helix content remain almost similar as reference. These properties show modulation among GI isolates. For example, while in Ishikawa and JEV28, GRAVY decline, net charge of Ishikawa and JEV45 show an increase. Although Ishikawa remains unaffected, coil to helix transition is apparent for both JEV28 and JEV45.

Homology modeling allows gaining structural insight from protein sequence [54] which further helps to extract structural information $[55,56]$ including prediction of conformational epitopes [57], localizing linear epitopes [42] and prediction of the effects of SNPs via thermodynamic cycle of folded and unfolded states of wild and mutant proteins [58]. In present study, we developed a total of 18 model structures (3 plus 15) for ecto domain of $E$ protein. The first three models are of $E$ protein of Ishikawa, JEV28 and JEV45 isolates. The rest 15 are models wherein each structure possesses single positional mutation (as in Table 1) with reference to the vaccine strain, SA14-14-2. While the former three are useful for general structural characterization of substitutions and epitopes, the later are crucial for understanding the effect of SNP on overall conformational stability of protein, and its disease association in relation to protein function. Homology models were obtained by the use of advance modules of Modeller v9.11 [24] followed energy minimization using CHARMM force field of NAMD package [26] in presence of explicit water as solvent with appropriate disulfide bond patching. Models thus obtained were evaluated as earlier procedures [20-23]. Topology of each model was compared with that 
Table 1 List of domain specific mutations in E protein of Gl isolates in reference (Ref) to GIII strains

\begin{tabular}{|c|c|c|c|c|c|c|c|c|}
\hline \multirow[b]{2}{*}{ E Protein } & \multirow[b]{2}{*}{ Mutations } & \multicolumn{4}{|l|}{ Genotype I (Gl) } & \multicolumn{3}{|c|}{ Genotype III (GIII) } \\
\hline & & $\begin{array}{l}\text { AB051292 } \\
\text { [Japan, Ishikawa, 1994] }\end{array}$ & $\begin{array}{l}\text { JN703381 } \\
{[W B, J E V 28 \text { 2010] }}\end{array}$ & $\begin{array}{l}\text { JN703382 } \\
{[W B, J E V 212010]}\end{array}$ & $\begin{array}{l}\text { KC526872 } \\
{[W B, J E V 45 \text { 2011] }}\end{array}$ & $3 \mathrm{P} 54$ & $\begin{array}{l}\text { SA14 } \\
\text { virulent } \\
\text { M55506 } \\
\end{array}$ & $\begin{array}{l}\text { SA14-14-2 } \\
\text { Non-virulent } \\
\text { JN604986 } \\
\end{array}$ \\
\hline \multirow[t]{7}{*}{ Domain I } & $\mathrm{N}-2 \mathrm{H}$ & . & + & + & + & $N$ & $N$ & $N$ \\
\hline & $\mathrm{N}-2 \mathrm{~T}$ & + & . & . & . & $N$ & N & N \\
\hline & Y183F & + & . & . & . & Y & Y & Y \\
\hline & R193K & + & . & . & . & $\mathrm{R}$ & $\mathrm{R}$ & K \\
\hline & $\mathrm{K} 138 \mathrm{E}^{\mathrm{a}}$ & $(+)^{a}$ & $(+)^{\mathrm{a}}$ & $(+)^{a}$ & $(+)^{a}$ & $K^{b}$ & E & K \\
\hline & $\left.V 176\right|^{a}$ & $(+)^{a}$ & $(+)^{\mathrm{a}}$ & $(+)^{a}$ & $(+)^{\mathrm{a}}$ & $V^{b}$ & । & V \\
\hline & $\mathrm{A} 177 \mathrm{~T}^{\mathrm{a}}$ & $(+)^{a}$ & $(+)^{a}$ & $(+)^{a}$ & $(+)^{\mathrm{a}}$ & $\mathrm{T}$ & $\mathrm{T}$ & A \\
\hline \multirow[t]{9}{*}{ Domain II } & $C-60 Y$ & + & & . & . & $C$ & $C$ & C \\
\hline & N103K & + & + & + & + & N & N & $\mathrm{N}$ \\
\hline & $\mathrm{F} 107 \mathrm{~L}^{\mathrm{a}}$ & $(+)^{a}$ & $(+)^{\mathrm{a}}$ & $(+)^{a}$ & $(+)^{a}$ & $F^{b}$ & L & $\mathrm{F}$ \\
\hline & T129M & + & . & & . & $\mathrm{T}$ & T & T \\
\hline & A222S & + & + & + & + & A & A & A \\
\hline & G244E & + & + & + & + & G & G & G \\
\hline & G261S & . & + & + & + & G & G & G \\
\hline & $\mathrm{H} 264 \mathrm{Q}^{\mathrm{a}}$ & $(+)^{a}$ & $(+)^{a}$ & $(+)^{a}$ & $(+)^{\mathrm{a}}$ & Q & Q & $\mathrm{H}$ \\
\hline & $M 279 K^{a}$ & $(+)^{a}$ & $(+)^{a}$ & $(+)^{\mathrm{a}}$ & $(+)^{\mathrm{a}}$ & $M^{b}$ & K & M \\
\hline \multirow[t]{7}{*}{ Domain III } & V315A & + & + & + & + & V & V & V \\
\hline & S327T & + & + & + & + & S & S & S \\
\hline & A366S & + & . & & . & A & A & A \\
\hline & V372L & + & & + & + & V & V & V \\
\hline & M374I & + & . & + & + & M & M & M \\
\hline & G388K & + & & + & + & G & G & G \\
\hline & W396R & + & . & + & + & W & W & W \\
\hline \multicolumn{2}{|c|}{ Total mutations } & 21 & 13 & 17 & 17 & 4 & 6 & Ref \\
\hline \multicolumn{2}{|c|}{ Net mutations } & 15 & 7 & 11 & 11 & - & - & \\
\hline
\end{tabular}

Amino acids marked with ${ }^{\mathrm{b}}$ sign in 3P54 have been identified as mismatches between PDB and UNIPROT sequence (P27395)

All comparisons are made for first 406 residues of E protein (ecto domain). All three Gl isolates of Midnapur/WB are taken with earlier Japan strain (Ishikawa). In GIII category, three sequences such as 3P54_A, virulent SA14 (ID M55506) and attenuated non-virulent SA14-14-2 (ID JN604986) are considered. + Sign indicates presence and dot indicates absence of mutations respectively. (+) indicates category of reported neurovirulent mutations [13]. Identical neurovirulent mutations of present isolates as SA14 are identified with ${ }^{\text {a }}$ sign

of template by superposition of $\mathrm{C}_{\alpha}$ atomic coordinates and the average RMSD (Root mean square deviation) value was found to vary in the range 1.12-1.44 $\AA$. Absolute per residue sum of surface area by NACCESS [44] procedure for template is seen to be $40.1 \AA^{2}$ and that for models (Ishikawa, JEV28 and JEV45) vary between 42.9 and $43.7 \AA^{2}$. In the former, frequency of buried residues is seen to be 161 and that for models vary between 160 and 161 when threshold for core is set to $\leq 20 \AA^{2}$ value. Detailed comparison between template and models on salt bridges and its energetics were performed using our laboratory developed software [33-35]. Frequency of salt bridges (19 to 23) of models and side-chain specificity of their partners remain almost similar ( $\geq 75 \%)$ as template (data not shown).
Model structure of Ishikawa, JEV45 and JEV28 are presented in Fig. 3a, b and c respectively. Each of these structures highlights substituted residues (Table 1), disulfide bonds and domains (as DI, DII and DIII). Domain I is the middle domain that (Fig. 3, red colored region reconstituted by 127 residues in three stretches: $1-51,135-193$ and 283-299) possesses nine stranded $\beta$-barrel structure with two disulfide bonds $\left(C_{3}-C_{30}\right.$ and $\left.C_{190}-C_{287}\right)$ and model specific amino acid substitutions $(\mathbf{N} 2 \mathrm{H}, \mathbf{N} 2 \mathrm{~T}$, A177T, Y183F and R193K). It also contains the conserved glycosylation site (N154). Domain II (yellow colored) is the distal domain which forms extended structure of 172 residues constituted by two regions (52-134 and 194-282) of E protein. It is stabilized by 


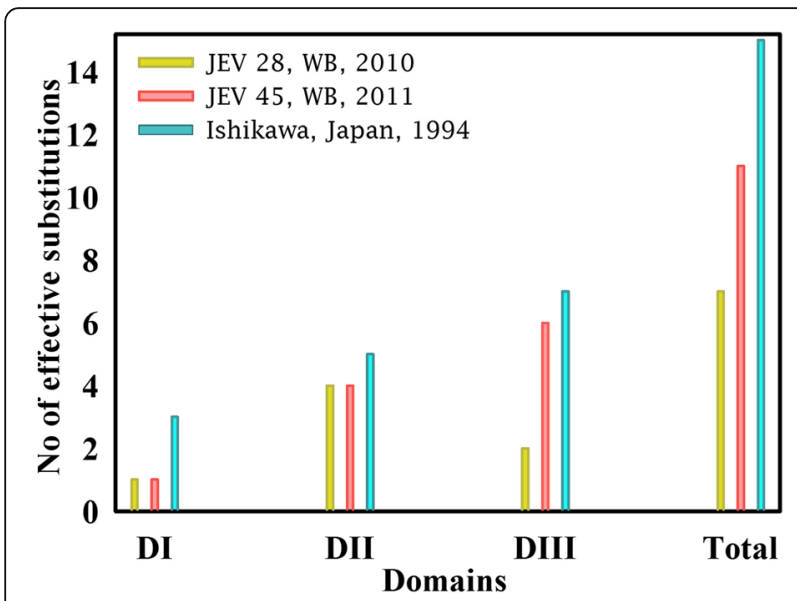

Fig. 1 Comparison of net substitutions for each domains and total of E protein among JEV28 (yellow), JEV45 (red) and Ishikawa isolates (cyan). JEV21 is not included in the plot as it is identical to JEV45

three disulfide bonds $\left(C_{60}-C_{116}, C_{92}-C_{121}\right.$ and $C_{74^{-}}$ $\left.C_{105}\right)$. This domain contains a total of seven substitutions (see Table 1) with respect to the template (C60Y, N103K, T129M, A222S, G244E, G261S and H264Q). Fusion loop which is 13 residues long is shown by purple color (at tip of Domain II) is needed for pathogenesis, infectivity and broad range antibody cross-reactivity $[52,59]$. It is highly conserved among all flaviviruses. Domain III (blue colored globular domain) of the model contains 100 residues (300-399) and forms typical immunoglobulin like fold at the $\mathrm{C}$-terminus of the ecto domain of $\mathrm{E}$ protein. This domain possesses one disulfide bond $\left(\boldsymbol{C}_{304}-C_{335}\right)$ and seven substitutions (V315A, S327T, A366S, V372L, M374I, G388K and W396R). In this domain a tripeptide sequence RGD

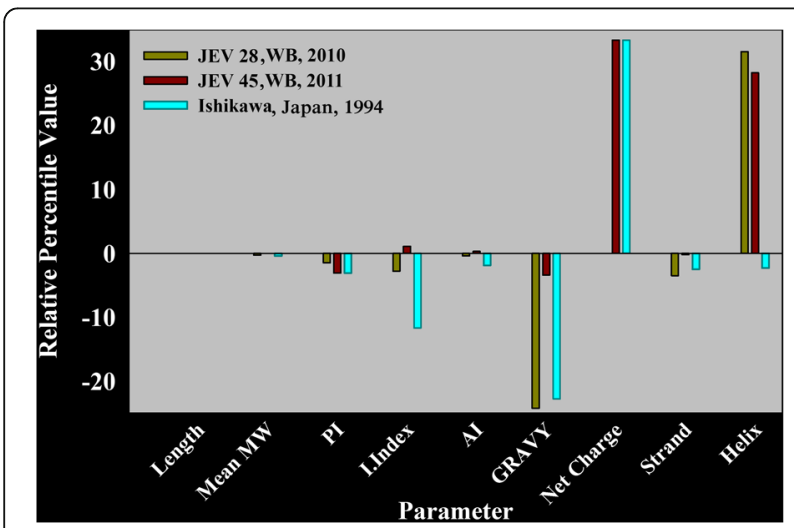

Fig. 2 Residual plot of average physicochemical properties for $\mathrm{Gl}$ isolates (Ishikawa, JEV28 and JEV45) in reference to SA14-14-2. Analyses were performed using PHYSICO [17] and PHYSICO2 [18] programs with an input of E proteins of SA14-14-2, Ishikawa, JEV28 and JEV45 in FASTA format. Percentile difference for each property was computed against the reference data and plotted using SYSTAT Sigma Plot v11.0
(387-389; blue colored) form a motif which is believed to play role in receptor interaction $[5,6,60]$.

Although all models were evaluated using multiple authentic procedures [20-23], results for the model JEV45 is shown in the Right panel of the Fig. 3. Energetic profile of the model (green trace) and the template (red trace) are seen to be almost identical when plotted as a function of residue position as obtained by ANOLEA [61] (Fig. 3: D1) and VERIFY3D analysis [31] (Fig. 3: D2). Ramachandran plot for main chain dihedral angles and PROCHECK analysis [29] (Fig. 3: D3 and D4 respectively) show amino acid residues, occupying core (92\%) and allowed (8\%) regions.

\section{Disease relation of substitutions}

There are a maximum of 15 substitutions for GI isolates apart from six reversal type (Tables 1 and 2). Are all these substitutions lethal? How could they be related with protein function and disease association? To resolve this, we present results of sequence and structure based prediction of the effect of these SNPs in Table 2. Sequence based prediction identify fatal substitutions as $\mathrm{D}$ and normal as $\mathrm{N}$ based on score. Structure based method computes overall conformational free energy change $\left(\Delta \Delta G_{W T \rightarrow M u}\right)$ in $\mathrm{Kcal} \mathrm{mol}^{-1}$. These results (Table 2 ) show that only 4 of 15 mutations are lethal, disease associated and cause protein malfunctioning. Rest 11 substitutions are normal and non-disease associated. In this group of mutations, $+0.50 \leq \Delta \mathbf{\Delta} \leq+2.00$ and $-2.00 \leq \Delta \Delta \mathbf{G} \leq-.50$ categories are also observed.

One of the fatal substitutions (from sequence based methods) i.e. N103K is seen to be common in all GI isolates (Table 1). It is present in the fusion loop region (Fig. 3) which is known to initiate host-virus interaction and eventual viral entry. Two of the fatal substitutions i.e. G388K and W396R are common for JEV21, JEV45 and Ishikawa but absent in JEV28. Both these substitutions are present in antigenic domain III of E protein (Fig. 3). The substitution C60Y is only present in Ishikawa/Japan isolate but not in any of the WB isolates. Notably C60 is involved in the formation of disulfide bond in domain II. Unlike normal, these 4 fatal substitutions show high change of overall conformational free energy of which G388K and N103K are positive and that in case of W396R and C60Y are negative.

\section{Epitope prediction}

Envelope glycoprotein of JEV is 500 amino acids long of which ecto domain constitutes about 406 residues. The protein has been the major focus for immunoinformatics studies for its neutralizing activity and antigenic cross reactivity from different flaviviruses $[62,63]$. In fact clathrin-mediated viral internalization was reported to be guided by the protein. At present the only available 


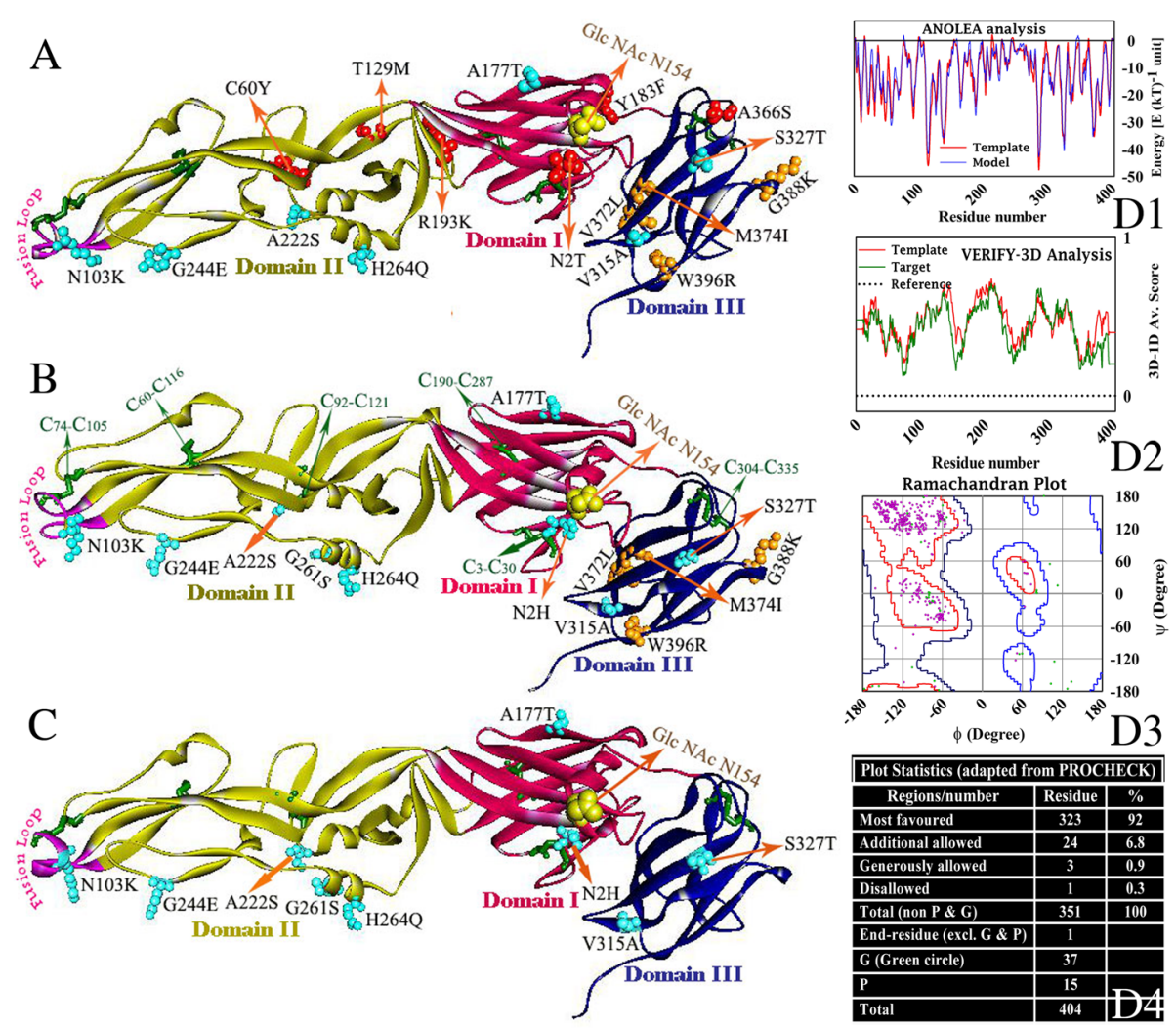

Fig. 3 Backbone Ca-traces of homology model (a for Ishikawa, b for JEV45 and c for JEV28) of the ecto domain of PM0080325, PM0080324 and PM0080323 respectively. Domain I (red), domain II (yellow) and domain III (blue) are shown in different colors. Fusion loop (purple) and substitutions with respect to the template (SA14-14-2) are highlighted in each of the model based on their occurrence (see Table 1). Disulfide bonds (green) are also highlighted in each of the model based on their occurrence and only labeled in case of JEV45. Comparison of ANOLEA-profiles [61] of PM0080324 (green trace) with template (red trace) (D1), VERIFY3D (D2) analysis [31] and Ramachandran plot (D3) of main chain dihedral angles (core region is outlined in deep blue and allowed region in red) for residues (glycine as green circle and non-glycine as pink points) of the model along with PROCHECK [29] analysis (D4) are presented for model validation

vaccine for prevention of JEV mediated AES/JE is derived from live or inactivated form of GIII strain SA1414-2. However, the efficacy of immunization with the current vaccine was questioned due to the fact that prevaccinated patients showed symptoms of JE/AES with co-circulation of GI strain in their serum [4, 10]. Such reports of emergence of GI strain from the pool of GIII in Asian countries signaling for design of high selective epitopes.

\section{B-cell epitope prediction}

B-cell epitopes are effective for induction of neutralizing antibody in relation to the viral entry. Identification and characterization of these epitopes would help in design of vaccine. B-cell epitopes having high prediction score, low model energy (i.e. high conformational stability), high average accessibility to the surface of protein and high average conservation were selected (Fig. 3). Our predicted epitopes (Table 3) show overlap with predetermined epitope segments [64]. 7 of 8 epitopes (Table 3) seen to harbor GI specific substitutions (Table 1) and four of these seven epitopes namely VEMEPPFGDSYIVVGRGDKQ, GWGKGCGLFGKGSIDTCAKF, H WHKAGSTLGKAFSTTLKGA and IEASQLAEVRSYYYHASVTD are seen to contain fatal substitutions.

Linear epitopes are predicted from sequence of $E$ protein [42]. Each epitope segment possesses specific folds or topology in three dimensional structure of the protein. We have localized all these linear epitopes in the structure of which the epitope GWGKGCGLFGKGSIDTCAKF is presented in Fig. 4 AII. It is seen that the epitope covering almost entire region of the fusion loop (residue 98-110) which is important for viral infectivity [52]. Further, it is exposed to the surface of the protein. Ab initio structural analyses of these epitopes are performed using PEP-FOLD method [43]. The model energy as obtained from this analysis is presented in Table 3. Negative value indicates stabilized structure (Table 3, column 4). Typical structures of selective fatal epitopes are also shown in the Fig. 4B I and II.

Although fatal mutations are seen to be populated in linear B-cell epitopes, their occurrence in conformational Bcell epitopes would be more relevant as sequentially distant 
Table 2 Sequence and structure based evaluation of the effect of mutations (Table 1)

\begin{tabular}{|c|c|c|c|c|c|c|c|c|c|c|}
\hline & \multirow{3}{*}{ Mutation } & \multicolumn{8}{|c|}{ Sequence based prediction } & \multirow{3}{*}{$\begin{array}{l}\text { Structure based prediction } \\
\mathrm{SDM} \\
\triangle \triangle \mathrm{G} \\
\mathrm{Kcal} \mathrm{Mol}{ }^{-1}\end{array}$} \\
\hline & & \multicolumn{2}{|l|}{ SIFT } & \multicolumn{2}{|c|}{ PHD-SNP } & \multicolumn{2}{|l|}{ SNAP } & \multicolumn{2}{|c|}{ META-SNP } & \\
\hline & & Score & $\overline{\text { Effect }}$ & Score & $\overline{\text { Effect }}$ & Score & $\overline{\text { Effect }}$ & Score & $\overline{\text { Effect }}$ & \\
\hline \multirow[t]{6}{*}{ Domain I } & $\mathrm{N} 002 \mathrm{H}$ & 0.500 & $\mathrm{~N}$ & 0.056 & $\mathrm{~N}$ & 0.180 & $\mathrm{~N}$ & 0.115 & $\mathrm{~N}$ & +0.01 \\
\hline & $\mathrm{K} 138 \mathrm{E}^{\mathrm{a}}$ & 1.000 & N & 0.121 & N & 0.185 & $\mathrm{~N}$ & 0.111 & N & +0.52 \\
\hline & $\mathrm{V} 1761^{\mathrm{a}}$ & 0.400 & N & 0.047 & N & 0.160 & $\mathrm{~N}$ & 0.090 & N & +0.22 \\
\hline & $\mathrm{A} 177 \mathrm{~T}^{\mathrm{a}}$ & 1.000 & N & 0.042 & N & 0.105 & N & 0.063 & N & $-0.17^{b}$ \\
\hline & Y183F & 0.200 & $\mathrm{~N}$ & 0.488 & N & 0.525 & $\mathrm{~N}$ & 0.367 & N & +1.79 \\
\hline & R193K & 0.450 & N & 0.490 & N & 0.340 & N & 0.414 & N & -0.81 \\
\hline \multirow[t]{9}{*}{ Domain II } & CO60Y & 0.000 & $\mathrm{D}$ & 0.935 & $\mathrm{D}$ & 0.800 & D & 0.882 & $D$ & -2.33 \\
\hline & N103K & 0.000 & $D$ & 0.833 & $D$ & 0.780 & D & 0.771 & D & +1.61 \\
\hline & $\mathrm{F} 107 \mathrm{~L}^{\mathrm{a}}$ & 1.000 & $N$ & 0.218 & $N$ & 0.310 & $\mathrm{~N}$ & 0.155 & $\mathrm{~N}$ & -0.97 \\
\hline & $\mathrm{T} 129 \mathrm{M}$ & 0.070 & $N$ & 0.106 & $N$ & 0.410 & $N$ & 0.143 & $N$ & +0.86 \\
\hline & A222S & 0.420 & $N$ & 0.177 & $N$ & 0.115 & $N$ & 0.162 & N & -0.77 \\
\hline & G244E & 1.000 & $N$ & 0.070 & $N$ & 0.215 & $N$ & 0.065 & $N$ & +1.85 \\
\hline & G261S & 0.200 & $N$ & 0.152 & $N$ & 0.105 & $\mathrm{~N}$ & 0.134 & $N$ & +1.74 \\
\hline & $\mathrm{H} 264 \mathrm{Q}^{\mathrm{a}}$ & 0.074 & $\mathrm{~N}$ & 0.074 & $N$ & 0.225 & $\mathrm{~N}$ & 0.078 & $\mathrm{~N}$ & $-0.75^{b}$ \\
\hline & $\mathrm{M} 279 \mathrm{~K}^{\mathrm{a}}$ & 0.390 & N & 0.159 & N & 0.255 & N & 0.121 & N & -1.97 \\
\hline \multirow[t]{7}{*}{ Domain III } & V315A & 0.620 & $N$ & 0.066 & $N$ & 0.400 & $\mathrm{~N}$ & 0.094 & $N$ & -1.63 \\
\hline & $\mathrm{S} 327 \mathrm{~T}$ & 0.820 & $N$ & 0.067 & $N$ & 0.175 & $\mathrm{~N}$ & 0.103 & $N$ & +0.97 \\
\hline & A366S & 0.740 & $\mathrm{~N}$ & 0.190 & $N$ & 0.235 & $\mathrm{~N}$ & 0.185 & $\mathrm{~N}$ & -1.18 \\
\hline & V372L & 0.310 & $\mathrm{~N}$ & 0.129 & $N$ & 0.245 & $\mathrm{~N}$ & 0.164 & $\mathrm{~N}$ & -0.79 \\
\hline & M374I & 0.540 & $N$ & 0.225 & $N$ & 0.365 & $\mathrm{~N}$ & 0.169 & $N$ & +0.08 \\
\hline & G388K & 0.010 & $\mathrm{D}$ & 0.910 & $\mathrm{D}$ & 0.775 & D & 0.793 & D & +3.00 \\
\hline & W396R & 0.000 & D & 0.841 & D & 0.865 & D & 0.819 & D & -2.48 \\
\hline
\end{tabular}

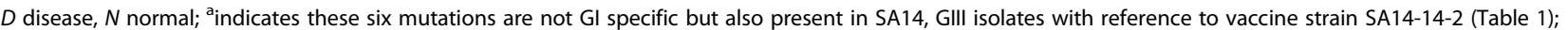
bindicates $\Delta \Delta \mathrm{G}$ was calculated in reverse mutation form i.e. T177A and Q264H as WT E protein possesses T and Q at these positions respectively Server based four independent methods for sequence of ecto domain of E protein and Site Directed Mutator (SDM) [41] method for structure of all isolates was used for the purpose (see Materials and Methods for details)

residues form a three dimensional shape for binding a receptor [65]. We presented conformational epitope in Fig. 4 AI. It is seen that residues forming conformational epitopes are localized at three distinct region of $\mathrm{E}$ protein: two in domain II and one in domain III. The fatal mutations are seen to fall in these regions with very high specificity $(\geq 80 \%)$. Interestingly three of four fatal mutations (N103K, G388K and W396R) are populated both in linear (Table 3) and conformational epitopes (Fig. 4 AI). Further, in the later case, mutant residue has been a part of one contiguous stretch instead of distantly and singly spaced residues coming in close proximity. Do these fatal mutations in peptideepitopes affect overall topology? To check this we compared 3D structures of wild-type and mutant-type peptideepitopes (Fig. $4 \mathrm{BI}$ and II). It is seen that the later undergoes large conformational change relative to the earlier as revealed by their RMSD values (Fig. $4 \mathrm{BI}$ and II). RMSD of 388K.pdb $(\mathrm{MU})=8.2 \AA$ w.r.t. 388G.pdb (WT) and RMSD of 396R.pdb (MU) $=10.6 \AA$ w.r.t. 396W.pdb (WT).

\section{T-cell epitope prediction}

In adaptive immune response, $\mathrm{T}$-cells mediated proliferation, secretion of cytokines (that stimulate antibody production by B-cells) and apoptosis are resulted when a ternary complex of (MHC-I/II)-(peptides)-(TCR$\left.\mathrm{CD} 8^{+} \mathrm{Tc} / \mathrm{CD} 4^{+} \mathrm{Th}\right)$ is formed. T-cell-mediated immunity is essential for controlling infection of a variety of mammalian cells by flaviviruses. During viral infection, the expression of both MHC-I and MHC-II molecules increases and the functional $\mathrm{CD}^{+} \mathrm{T}$ cells provide protection by direct viral clearance whereas $\mathrm{CD} 4^{+} \mathrm{T}$ cells provide protection by eliciting protective antibody responses as well as by generating both B cell and CD8+ $\mathrm{T}$ cell memory responses. Flavivirus $\mathrm{E}$ protein is the major target of immune responses and has been shown to use both for MHC-I/CD8 ${ }^{+} \mathrm{T}$ cell as well as MHC-II/ $\mathrm{CD}^{+} \mathrm{T}$ cell specific epitope prediction $[62,66-69]$. In this study, we use IEDB Analysis Resource v2.14 [46] for prediction of T-cell epitopes. 
Table 3 B cell specific antigenic peptide epitopes short listed from a large set of initial population based on their antigenic score, model energy, average ASA (Accessible surface area) and average conservation

\begin{tabular}{|c|c|c|c|c|c|}
\hline $\begin{array}{l}\text { Start } \\
\text { Position } \\
\end{array}$ & $\begin{array}{l}\text { Linear } \\
\text { Epitope }\end{array}$ & Score & $\begin{array}{l}\text { Model } \\
\text { Energy }\end{array}$ & $\begin{array}{l}\text { Av. } \\
\text { ASA }\end{array}$ & $\begin{array}{l}\mathrm{Av} . \\
\mathrm{H}\end{array}$ \\
\hline 25 & ELTYSGSDGPCKIPIVSVAS & 0.986 & -23.71 & 22.8 & 0.01 \\
\hline 32 & VEMEPPFGDSYIWGRGDKQ & 0.977 & -24.54 & 37.1 & 0.02 \\
\hline 100 & GWGKGGGLFGKGSIDTCAKF & 0.956 & -29.81 & 42.1 & 0.02 \\
\hline 9 & STVARCPTTGEAHNEKRADS & 0.926 & -12.95 & 59.3 & 0.01 \\
\hline 395 & HWWHKAGSTLGKAFSTTLKGA & 0.876 & -2 & 97.2 & 0.02 \\
\hline 21 & CTSKAIGRMIQPENIKYEVG & 0.854 & -18.21 & 38.2 & 0.06 \\
\hline 349 & TPVGRLVTVNPFVATSSSINS & 0.923 & -20.55 & 39.9 & 0.04 \\
\hline 48 & IEASQLAEVRSY $\underline{Y} Y H A S V T D$ & 0.967 & -32.65 & 31.4 & 0.06 \\
\hline
\end{tabular}

NACCESS program [44] was used to generate residue accessibility data. Then average accessibility was computed using side chain relative accessibility values. Variability was calculated using full length $E$ protein sequences (total 50) and positional Shannon entropy $(\mathbf{H})[17,18]$ was computed for each homologous position. The Av. Variability is the average of all residues value. Average is made up to 404 residues as the model has 404 residues like template

Three score criteria were followed for screening of these peptide epitopes: a] MHC-I binding score, b] proteosomal cleavage score and c] antigenicity score. MHC-I presents endogenous peptide epitopes to the $\mathrm{CD}^{+}$cytotoxic $\mathrm{T}$ cell receptor (TCR). Table 4 presents predicted MHC-I (also known as HLA in human) endogenous peptides of $\mathrm{E}$ protein. HLA-restriction of each peptide was achieved by setting a lower cutoff for binding score $(\leq 20)$ and hence better complex formation of peptide with respective allele of HLA is expected. Highly screened peptide epitopes are seen to harbor JEV genotype-I specific lethal substitutions (Table 2) which are pointed out by bold fonts with underline. The epitope YIVVGRKDK is most antigenic and harboring the G388K lethal substitution (Table 4). The alleles, with which it associates are HLA-A*03:01, HLA$\mathrm{A}^{* 11: 01}$ and HLA-A*68:01 which are known to populate among Asian population [70]. Similar details are presented for other epitopes.

JEV E protein was also used for prediction of MHC class II specific epitopes using the above server. MHC-II presents exogenous peptide epitopes to the CD4+ T-helper cell (Th) receptor (TCR). As MHC-II presents exogenous epitope peptides, cleavage score is not available in such prediction (Table 5). Like MHC-I, GI specific lethal substitutions are also shown in the selected peptide epitopes. Allelic association against each epitope is achieved by setting lower cutoff for MHC II binding. Lower binding score is the indicator of better complex formation between peptide epitope and MHC molecule. Highest antigenicity score and very low MHC- II binding score is observed for the epitope GFTDRGWGKGCGLFG which associates mostly DRB1 class of alleles of type 3, 8 and 11 .

Prediction of T-cell epitopes are performed using sequence of E protein in IEDB server [46]. How these epitopes are structurally compatible in interaction with peptide binding groove (PBG) of their corresponding MHC molecules? To check this we evaluated energetics of docked-complex of epitope. We further checked positional Shannon conservation $[17,18]$ and average sidechain accessibility [44] of these peptide epitopes. Table 6 presents average conservation (Table 6: column 5), accessibility (column 6), epitope conformational stability (column 7) and binding affinity for the PBG of MHCs (column 8 through 11). When positional Shannon entropy (with BLOCK length $\geq 70$ ) $\leq 0.50$, those positions are taken as conserve. In our case, observed variability for epitopes are seen to be far less and even zero (column 5). All

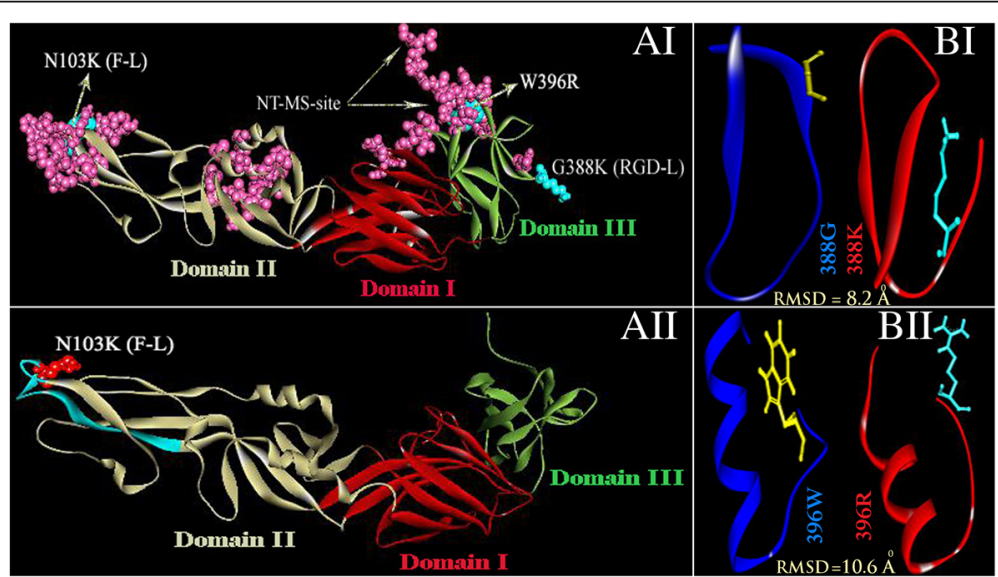

Fig. 4 B cell specific conformational antigenic determinants (Al) and linear epitopes (All). (Al, All) In each case domains of E protein are presented by different colors (i.e. domain I red, domain II silver and domain III green). (B) Representative Epitope pairs (BI for G388K and BII for W396R) for comparison of main chain topology of wild-type (blue) and mutant-type (red). The wild-type (yellow) and mutant (cyan) type residues are shown in stick formats. Each of this peptide structure was generated using PEP-FOLD [43] followed by normalization and fixation of charge and potential. RMSD of mutant structure was computed in reference to its wild-type. F-L: Fusion loop; RGD-L: RGD loop 
Table 4 Prediction of MHC class I specific peptide epitopes

\begin{tabular}{|c|c|c|c|c|c|}
\hline Start & Peptide & $\begin{array}{l}\text { MHC-I } \\
\text { binding } \\
\text { score }^{a}\end{array}$ & $\begin{array}{l}\text { Proteosomal Cleavage } \\
\text { score }^{\text {b }}\end{array}$ & $\begin{array}{l}\text { Allele } \\
\text { (Human Leukocyte Antigen [HLA] specific) }\end{array}$ & Antigenic Score ${ }^{c}$ \\
\hline 59 & YYYYHASVTD & $1.3-17.0$ & 0.82 & $\begin{array}{l}\text { HLA-A*24:02,HLA-A*23:01,HLA-A*29:02,HLA-B*18:01,HLA-B*39:01, } \\
\text { HLA-B*48:01,HLA-B*51:01,HLA-C*01:02,HLA-C*03:03,HLA-C*06:02, } \\
\text { HLA-C*07:01,HLA-C*07:02,HLA-C*07:04,HLA-C } 12: 02, H L A-C^{*} 14: 02, \\
\text { HLA-C*16:01,HLA-G*01:01,HLA-G*01:02,HLA-G*01:03,HLA-G*01:04, } \\
\text { HLA-G*01:06 }\end{array}$ & 0.9572 \\
\hline 95 & GFTDRGWGK & $4.4-15.5$ & 0.85 & $\begin{array}{l}\text { HLA-A*03:01,HLA-A*11:01,HLA-A*29:02,HLA-A*30:01,HLA-A*33:03, } \\
\text { HLA-A*31:01,HLA-A*68:01,HLA-A*74:01,HLA-C*04:01 }\end{array}$ & 1.4597 \\
\hline 382 & YIWGRKDK & $6.4-6.6$ & 0.92 & HLA-A*03:01; HLA-A*11:01;HLA-A*68:01 & 2.5185 \\
\hline 394 & HHRHKAGST & $8.9-13.7$ & 0.70 & HLA-B*07:02, HLA-B* 14:02 & 0.5088 \\
\hline
\end{tabular}

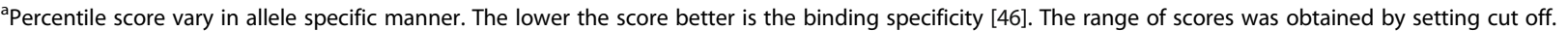
${ }^{b}$ Proteosomal cleavage score is the average of two kinds of scores: constitutively expressed type and INF- $\gamma$ induced type. ${ }^{\mathrm{C}}$ Antigenic score was computed using Doytchinova and Flower's (2007) method [47]

Three score criteria were followed to screen the epitopes: MHC class I binding score, proteosomal cleavage score and antigenicity score. Association of HLA alleles for each epitope was determined by a cut off for percentile rank value $\leq 20.0$. Epitopes were further checked for their efficacy in interactions at the peptide binding groove (see below)

epitopes are seen to be solvent exposed as their average accessibility is seen to be $>20$ (column 6). Peptides structural parameter i.e. conformational stability is seen to be negative (column 7). However, greater stability for MHC II specific peptides seems to be related with sizes of peptide epitopes. Docking based energetics analyses are presented in column 8 through 11 (Table 6) are for overall stability (GE), Van der Waals (VDW), atomic contact energy (ACE) and hydrogen bonding (HB) interactions in Kcal $\mathrm{Mol}^{-1}$ respectively. All these energy of interactions are highly negative and hence stabilizing.

To check the interaction of epitopes with the peptidebinding-groove (PBG) of MHC class I and MHC class II representatives, typical complexes for each class is presented in the Fig. 5. Figure shows two views from each class specific interaction along with mutated residue (white), peptide binding site (PBG). PBG is formed by two domains ( $\alpha 1$ and $\alpha 2$ ) of $\alpha$-chain (homo dimer) in
MHC - I, in MHC-II it is hetero dimer and formed by $\alpha 1$ and $\beta 1$ domains of $\alpha$ and $\beta$ chains respectively.

\section{Diversity in T-cell epitopes}

All T-cell epitopes (Tables 4 and 5) are conserved in nature as judged by their positional Shannon Entropy values. These selected epitopes of GI strain of JEV from WB (Midnapur) and Japan harbor fatal substitutions namely: C060Y, N103K, G388K and W396R. How these epitopes are evolved in other geographical locations? To check the diversity in these epitopes a total of $613 \mathrm{E}$ protein sequences were grouped into 8 geographical regions (China-228, Japan-113, Taiwan-79, India-66, Korea-35, Thailand-33, Indonesia-27 and Vietnam-32) and subjected for IEDB-Conservancy analysis. The result is presented in (Additional file 1) for MHC - I and (Additional file 2) for MHC - II epitopes. Following points are noteworthy from the observation: i] for all geographical regions

Table 5 MHC class II specific top scored antigenic peptides along with associated alleles

\begin{tabular}{|c|c|c|c|c|}
\hline Start & Peptide & $\begin{array}{l}\text { MHC-II } \\
\text { binding } \\
\text { score }^{\mathrm{a}}\end{array}$ & $\begin{array}{l}\text { Allele } \\
\text { (Human Leukocyte Antigen [HLA] specific) }\end{array}$ & Antigenic Score \\
\hline 55 & EVRSYYYYHASVTDIS & $0.25-1.76$ & $\begin{array}{l}\text { DRB1*03:05,DRB1*04:01,DRB1*04:08,DRB1*04:09,DRB1*04:07,DRB1*04:21, } \\
\text { DRB1*04:17,DRB1*04:26,DRB1*04:62,DRB1*04:69,DRB1*04:74,DRB1*04:82, } \\
\text { DRB1*07:03,DRB1*07:01,DRB1*08:04,DRB1*09:07,DRB1*09:04,DRB1*09:01, } \\
\text { DRB1*09:09,DRB1*11:02,DRB1*11:21,DRB1*13:22,DRB1*15:06,DRB1*15:02 }\end{array}$ & 0.9127 \\
\hline 95 & GFTDRGWGKGGGGFG & $5.37-17.95$ & $\begin{array}{l}\text { DRB1*01:02,DRB1*03:05,DRB1*07:03,DRB1*08:01,DRB1*11:01,DRB1*11:28, } \\
\text { DRB1*13:05,DRB1*13:21,DRB1*15:02,DRB1*15:06,DRB5*01:05 }\end{array}$ & 0.9232 \\
\hline 380 & DSYIWGRKDKQINH & $0.1-1.97$ & $\begin{array}{l}\text { DRB1*03:06,DRB1*03:05,DRB1*03:01,DRB1*03:08,DRB1*03:07,DRB1*08:01, } \\
\text { DRB1*08:04,DRB1*08:06,DRB1*11:01,DRB1*11:07,DRB1*13:07,DRB1*13:04, } \\
\text { DRB5*01:01,DRB5*01:05 }\end{array}$ & 1.5861 \\
\hline 383 & IWGRKDKQINHHR & $0.4-4.04$ & $\begin{array}{l}\text { DRB1*03:06,DRB1*03:05,DRB1*03:01,DRB1*03:08,DRB1*03:07,DRB1*04:02, } \\
\text { DRB1*08:01,DRB1*08:04,DRB1*08:06,DRB1*08:13,DRB1*11:02,DRB1*11:07, } \\
\text { DRB1*1 } 1: 21, \mathrm{DRB} 1 * 13: 01, \mathrm{DRB} 1 * 13: 04, \mathrm{DRB} 1 * 13: 27, \mathrm{DRB} 1{ }^{*} 13: 22\end{array}$ & 1.1724 \\
\hline
\end{tabular}

${ }^{\mathrm{a}}$ Percentile score vary in allele specific manner. The less the score better is the binding. The range of scores was obtained by setting cut off. ${ }^{\mathrm{b}} \mathrm{Antigenic}$ score was computed using Doytchinova and Flower's (2007) method [47]

Epitopes were further checked for their efficacy in interactions at the peptide binding groove (see below) 
Table 6 Energetics of docking complex formed between epitopes and PBG of MHCs along with their conservation, accessibility and conformational stability values

\begin{tabular}{|c|c|c|c|c|c|c|c|c|c|c|}
\hline \multicolumn{4}{|c|}{ IEDB Server Analyses } & \multirow{2}{*}{$\begin{array}{l}\text { Shannon } \\
\text { Variability } \\
\text { Average } \\
\text { Shannon Entropy }\end{array}$} & \multirow{2}{*}{$\begin{array}{l}\text { NACCESS } \\
\text { Analysis } \\
\text { Average side-chain } \\
\text { Accessibility }\end{array}$} & \multirow{2}{*}{$\begin{array}{l}\text { Peptide } \\
\text { Analysis } \\
\text { CGE }\end{array}$} & \multicolumn{4}{|c|}{$\begin{array}{l}\text { Docking Analyses of } \\
\text { MHCs-Peptides } \\
\left(\text { Energy in Kcal Mol}{ }^{-1} \text { ) }\right.\end{array}$} \\
\hline & $\begin{array}{l}\text { SL. } \\
\text { NO. }\end{array}$ & $\begin{array}{l}\text { Amino acid } \\
\text { Position }\end{array}$ & Peptide & & & & $\overline{G E}$ & VDW $^{a}$ & ACE & $\mathrm{HB}$ \\
\hline \multirow[t]{4}{*}{ MHC-I } & 1 & 59 & $\underline{Y} \underline{Y} Y H A S V T D$ & 0.02 & 23.5 & -4.70 & -42.89 & -16.11 & -5.89 & -4.74 \\
\hline & 2 & 95 & GFTDRGWGK్ & 0.03 & 50.4 & -6.61 & -28.87 & -21.69 & -4.29 & -2.42 \\
\hline & 3 & 382 & YIWGRKDK & 0.01 & 32.0 & -5.52 & -29.36 & -11.13 & -4.13 & -1.95 \\
\hline & 4 & 394 & HHRHKAGST & 0.03 & 64.3 & -5.84 & -39.03 & -14.41 & -2.02 & -7.97 \\
\hline \multirow[t]{4}{*}{ MHC-II } & 1 & 55 & EVRSY $\underline{Y} Y H A S V T D I S$ & 0.04 & 24.7 & -16.96 & -48.65 & -15.18 & -9.98 & -2.92 \\
\hline & 2 & 95 & GFTDRGWGKGGCGLFG & 0.02 & 57.9 & -17.23 & -46.57 & -14.25 & -5.75 & -4.75 \\
\hline & 3 & 380 & DSYIWGRKDKRINH & 0.04 & 34.9 & -16.07 & -53.97 & -22.96 & -1.11 & -5.02 \\
\hline & 4 & 383 & 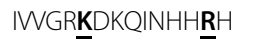 & 0.00 & 36.8 & -15.34 & -52.90 & -13.06 & -3.63 & -2.61 \\
\hline
\end{tabular}

${ }^{a}$ VDW is the net stabilization due to Van der Waals interactions i.e. (attractive VDW - repulsive VDW); GE global stabilization energy, ACE atomic contact energy, $H B$ hydrogen bond, CGE coarse-grained free-energy of peptide-structure used for docking [49]

and for all epitopes, large proportion of these peptide epitopes (Tables 4 and 5) remain similar as GIII type (SA14-14-2), ii] observed SNPs in these epitopes (namely: C060Y, N103K, G388K and W396R) in studied GI-strains (JEV28, JEV21 and JEV45 in WB and in Ishikawa in JAPAN) are not unique but other types of SNPs are also seen. The GI strains of Midnapur/ $\mathrm{WB} /$ India are only examples (of all 65 Indian strains) that contain similar fatal SNPs as earlier Japan GI strain (Ishikawa), iii] these SNP harboring epitopes are mostly related with the genotypic transition from III to I and V/II. For example in China the epitope YCYHASVTD constitutes 221 WT (i.e. GIII) and 7 mutant types. Again, 6 of these mutant types are from GI and 1 is from GV, iv] frequency and position of SNP in these epitopes vary for geographical regions and v] finally GI isolates of Midnapur are seen to be similar to earlier Ishikawa/Japan type but vary greatly from other Indian and non-Indian types.

\section{Discussion}

In spite of the availability of vaccine, JE/AES cases and deaths are frequent in different districts of West Bengal state, India. Emergence of GI and its co-circulation with GIII was reported for the first time in the district of Midnapur, WB even when patients were preimmunized with SA14-14-2 [3, 4]. Clinical symptoms and severity for GI mediated infection vary greatly from that of GIII which might involve viral and host factors. E protein plays crucial role in viral infectivity, virulence, host tropism and antigenicity [12]. In this context the reason of this genotypic transition and the study in relation to the development of GI specific vaccine are to be worked out. We apply in silico procedure using $\mathrm{E}$ protein sequence and its 3-dimentional models i] to identify critical among all acquired substitutions, ii] to workout epitopes harboring these critical substitutions and iii] to gain insight into the development of vaccine with potential in controlling GI cases.

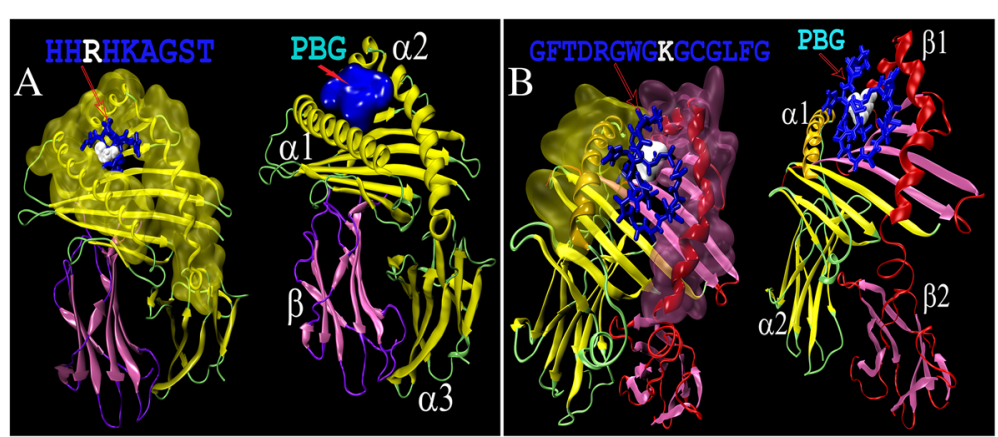

Fig. 5 Docking and structure based short listing of antigenic epitopes using human class I MHC (HLA-A i.e. 2X4O.pdb in a) and class II MHC (HLA-DRB1 i.e. 1DLH.pdb in b) crystal structures. Typical sequence and structure of docked peptide (blue color A \& B) with white one as mutated residue. PBG indicates peptide binding groove which was mapped using NACCESS procedure [44] (see Materials and Methods for details). Two views per MHC class are shown for better visualization of binding pocket and binding groove. Chains of both classes of MHCs are shown by conventional notations 


\section{Structural properties of models}

The E glycoprotein of JEV is 500 residues long of which 452 residues form extracellular domains and rest 48 residues form intra-membrane region (in part of 21, 6 and 21 residues). The protein has $\mathrm{pI}$ in the neutral range with net charge negative. It is predominantly $\beta$-sheeted. To gain insight into structure and mutations, we developed high precision models of GI isolates. Initial models are energy minimized to remove unwanted steric clash and to obtain global minimal structure. Multi procedure validations are done to assess quality. Low average RMSD (1.12-1.44 $\AA$ ) of models indicates almost identical main chain topology. Absolute residue-surface-area and frequency of coreresidues of models and template were almost identical. These assessments along with arrangement of secondary structures, specificity in di-sulfide bonds of models are indicative of well developed functional structure of the proteins. Although similar, there exists fine structural difference between models and the template. In template (from GIII) there are 33 strands of 209 residues (51\%) and that for JEV28 (GI), for example, is 33 strands of 225 residues (55\%). Similar difference also exists in other models which might have arisen due to genotypic variations due to substitutions (Table 1). Salt bridges are formed when side chains of acidic and basic residues are within $\leq 4 \AA$ distance [71]. Similar specificity $(\geq 75 \%)$ in these interactions in models and in template further indicates that the geometry and conformation of side chains are also well formed.

\section{Acquired GI specific substitutions and inducer of GIII to GI transition}

GI isolates JEV28 (also JEV21) and JEV45 was discovered from the Midnapur district of WB in 2010 and 2011 respectively. These strains were isolated as co-circulation cases in patient and were seen to be capable in escaping the effect of vaccination with SA14-14-2. These patients were thus developed JE clinical symptoms $[3,4,10,11]$. These isolates show similar locus specific mutations as Japan outbreak-GI-isolate (Ishikawa) in 1994. Our focus is to identify substitutions that may have relation with GIII to GI transition.

Mutations pertaining to neurovirulence and neuroinvasiveness seem to be genotype independent properties. These phenotypic properties depend on substitutions in E, other structural and non-structural proteins of JEV [72]. Cao QS, et al. (2011) [12] showed that the sequence of $E$ protein of their GI isolate is identical as virulent GIII strain (SA14) but differ from SA14-14-2 at 8 different positions (F107L, K138E, V176I, A177T, H264Q, M279K, V315A and $\mathbf{R 4 3 9 K}$ ) which they logically considered to be responsible for virulence phenotype of their isolate. Exactly similar results were also obtained both for GIII (JEV46 and JEV47) and GI (JEV28 and JEV45) isolates [4] indicating the observation is genotype independent. It is noteworthy that $\mathrm{E}$ protein of virulent (SA14) and non-virulent (SA14-14-2) strains differ by first 6 mutations (Table 1, marked with ${ }^{\text {a }}$ sign) [73]. Neuroinvasiveness could largely be related with the substitutions in other proteins but not in $\mathrm{E}$ protein. Nerome R, et al. (2007) and Cao QS, et al. (2011) [12, 13] reached to the same conclusion as far as neuroinvasiveness is concerned. Moreover, in reference to GP78/India [1] it was concluded that SA14-14-2 needs E244G, A315V and S366A for complete neurovirulence [72]. Nevertheless crucial substitutions that cause the transition of existing GIII to newly emerged GI (JEV28 and JEV45) in Midnapur district and allow escaping the effect of vaccination remained unanswered.

Table 1 compares substitutions in JEV28 and JEV45 (also JEV21; Ishikawa/Japan/1994) with non-virulent (SA14-142) vaccine strain (also virulent SA14; PDB ID 3P54). As mentioned above, mutations at positions: 138, 176 and 177 (in DI) and 107, 279 and 264 (in DII) emerge due to attenuation of virulent phenotype. SA14 and SA14-14-2 also differ at these positions (Table 1, mutations marked with $^{\mathrm{a}}$ sign). Additionally the ecto domain needs G244E, V315A and A366S substitutions for virulent phenotype (see above). It therefore indicates that these mutations are neither related to the transition of GIII to GI nor with the escape of vaccine effects. Thus to gain insight into the effect of homologous substitutions that may induce emergence of GI, we attempted rigorous sequence [37-40] and structure [41] based approaches using sequence and structure as input respectively. Out of all substitutions, only C60Y, N103K, G388K and W396R are GI specific and disease associated. As N103K is the only one which is common in all our GI isolates and showing disease association, it might be crucial for GIII to GI transition and escaping of GIII vaccine effect. It is present in the fusion loop region (98-110) which is crucial for viral infectivity [52, 59]. JEV45 (also JEV21) contains two additional GI specific fatal substitutions (G388K and W396R) that are absent in JEV28. C60Y is only present in Ishikawa strain. In silico site directed free energy evaluation [58] shows that C60Y and W396R are destabilizing whereas N103K and G388K are stabilizing. C60Y destroys one conserved disulfide bond (between $C_{60}-C_{116}$ ) and W396R causes removal of conserved Tryptophan residue. The stabilizing effect of N103K and G388K might be due to the side chain of Lysine that possesses a long flexible hydrocarbon tail with positive charge at its end which might facilitates stabilizing electrostatic interactions. Further $\mathrm{G}$ at $\mathbf{3 8 8}$ of DIII/E protein is present in the RGD motif $[6,60]$ important for cell-cell interaction. Substitution of such conserved glycine that imparts flexibility is known to have worst structural effect [74]. Overall, these 4 SNPs that are acquired in the course of viral evolution seems to be crucial for GI specific characteristics of $\mathrm{E}$ protein of which N103K acts as initiator for transition. 
Other substitutions such as N2H, N2T, T129M, A177T, Y183F, R193K, A222S, G244E, G261S, H264Q, V315A, S327T, A366S, V372L and M374I are nondisease associated. They could be divided into two categories: neutral and non-neutral-non-disease associated. While the former do not affect conformational properties of mutant protein the later does to some extent.

\section{B-cell epitopes harboring fatal substitutions and are crucial for neutralizing antibody}

Major challenge remains to understand the escape of neutralization of GI specific infection upon vaccination $[3,4]$. The purpose of vaccination is to induce proliferation of neutralizing antibody that in turn is expected to neutralize viral infection. The reports of co-circulation cases (GIII with GI in patients) in the district of Midnapur/ WB have been a serious concern. In this end the substitutions F107L, K138E, V176I, A177T, H264Q, M279K, G244E, V315A and A366S are unlikely to be responsible as i] these are related with virulent phenotype and ii] are observed both with GI and GIII isolates [4]. Prediction of neutralizing epitopes harboring fatal SNPs would not only help to understand escape mechanism but also be useful in designing GI specific vaccine that might take care possible GI-mediated outbreak in the region in near future. Our studies shows that of all SNPs only 4 such as C60Y, N103K, G388K and W396R are disease-associated of which former two are in domain-II and the later two are in domain-III of E protein which are the source of major antigenic determinants [53]. The question that are these SNPs present in our predicted population of B-cell epitopes. Bcell epitopes, both configurational (linear) and conformational (spatial), are thus predicted using authentic web servers. Initial population of both these epitopes is screened based on their scores. Linear epitopes were further screened for: a] accessibility, b] epitopes model energy and c] average conservation properties. It is interesting to note that these highly screened B-cell epitopes (Table 3) harbor disease associated SNPs namely C60Y, N103K, G388K and W396R. Of these four, N103K in fusion loop, G388K in RGD loop and W396R is in the N-terminal membrane attachment site (NT-MS site) (Fig. 4 AI). These regions are known to play important role for antigenicity and infectivity [75]. Their presence in both linear and conformational epitopes (in contiguous stretch of residues) indicates their importance in the development of vaccine. Large variations of RMSD of GI specific lethal mutation harboring peptide epitopes, point to the shift of wild-type (GIII specific) specificity in the neutralization reaction.

The only lethal substitution $\mathbf{N 1 0 3 K}$ is present in Midnapur/WB/JEV28 whereas Midnapur/WB/JEV21 and Midnapur/WB/JEV45 contain N103K, G388K and W396R. All these three isolates of Midnapur are newly emerged as GI [3]. Sarkar et al. (2013) interpreted the mutation G388K to be responsible "to escape from antibody neutralization or neutralizing epitope" based on physiochemical property and $\mathrm{IC}_{50}$ score for $\mathrm{MHC}-\mathrm{I}$ specific T-cell epitopes without confirming the presence of the residue in B-cell epitopes. In fact B-cell epitopes were not determined in the work. The authors have applied similar approach for the substitution N103K. The fact that substitutions at homologous positions could be acquired either by natural selection that affects protein function [76] or by genetic drift that imparts neutral effect [77] understanding the effects of substitutions on protein function need careful criteriabased evaluation. It may further be essential to confirm the presence of these substitutions on epitope for understanding escape mechanism. However, apart from the above linear epitopes, conformational B-cell epitopes (Fig. 4 AI) which are seen to be localized in most antigenic domains (DII and DIII) of the protein are seen to contain these above mentioned fatal SNPs with very high scores. The fact that each of these SNPs is seen to affect overall conformational stability of mutated E protein (Table 2) the possibility of altered specificity in neutralization reaction could not be ruled out. Taken together, it could be said that observation of repeated co-infection cases [3, 4] might be due to altered specificity of antigenic determinants harboring these fatal SNPs. These epitopes would also be useful for designing GI specific vaccine.

\section{Fatal substitutions in T-cell epitopes}

T-cell mediated immune response plays crucial role in proliferation of both cytotoxic T-lymphocytes and cytokines induced B-cell antibodies [78]. Length of $\mathrm{MHC}$ class I specific epitopes are 8 to 11 amino acids long and that for MHC class II is 13 to 17 amino acids long [78]. Unlike B-cell epitopes, selection of T-cell ones are MHC classes and alleles specific. We selected only four epitopes from each class following strict cutoff for MHC binding, proteosomal cleavage (for MHC class I only) and antigenicity score. Of all MHC class I epitopes, YIVVGRKDK possesses highest antigenicity and proteosomal cleavage score. It contains the lethal substitution G388K in its RGD motif which is known for host cell interaction $[6,60]$. The substitution, present in Midnapur (JEV45 and JEV21) and Japan isolates (Ishikawa), is shown to affect overall conformational stability of E-protein. Thus it may affect T-cell proliferation and cytokine mediated B-cell antibodies production. Interestingly the same substitution G388K is also present in MHC class II specific epitope DSYIVVGRIKDKQINH with very high binding and antigenicity score. The first GI isolate (JEV28) from Midnapur/WB contains only one lethal substitution i.e. N103K. It is present in the fusion loop of the $\mathrm{E}$ protein which is important for viral infectivity. Our 
predicted MHC class (I and II) specific epitopes has been GFTDRGWGK and GFTDRGWGKGCGLFG respectively that contains the said mutation. The former has higher specificity (lower binding score and higher antigenicity score). The fact that the patients, from which these strains were isolated, were reported to escape the neutralizing effect of GIII specific vaccine [4], these set of B-cell (see above) and T-cell epitopes can be used as reference for development of GI specific vaccine. Moreover other predicted epitopes that harbor lethal substitutions (such as C60Y and W396R) are also potential to alleviate GI specific danger $[3,4]$ from the district of Midnapur (see below).

Major (A, B, C) and minor (G) class I type HLA alleles that are associated with our selected epitopes are major alleles for zoonotic viral infection [79]. Similarly, haplotypes DRB1*04, DRB1*03, DRB1*08, DRB1*09 and DRB1*11 are abundant in our class II specific epitopes. These haplotypes were seen to be associated with zoonotic viral infection $[80,81]$.

The selected B-cell and T-cell epitopes of GI isolates of Midnapur/WB (JEV28, JEV21 and JEV45) are compared with isolates from other geographical regions such as China, Japan, Taiwan, Korea, Thailand, Vietnam and Indonesia (see Additional files 1 and 2). The mutation patterns of these epitopes are seen to be similar as Ishikawa/Japan but differ for isolates from other geographical regions. The mutated populations of these epitopesegments are very low $(\leq 2 \%)$ and are mostly related with genotypic transition from GIII to GI/GV/GII. For example of 228 isolates from China for the epitope YCYHASVTD, 220 are of GIII type, 7 are GI and 1 is GV type (see Additional file 1). Epitope-segments occupy different parts of E protein sequence that may have differential selection pressure from different geographical regions. For example the epitope YIVVGRGDK (382-390) shows more variants in Vietnam (7 of 32) than China (4 of 228) isolates and no variations are seen in Thailand isolates (0 in 33).

\section{Conclusions}

Our studies employ wide range of sequence and structure based in silico procedures on E protein of GI isolates from Midnapur/WB to understand GI specific lethal substitutions, its transition from the pool of GIII strain of JEV and prediction of B-cell and T-cell epitopes along with their diversity in Asian continent. Nine substitutions are commonly seen in the ecto domain of $\mathrm{E}$ protein of GI and GIII isolates are largely act as determinant of virulent phenotype of these GI isolates. 4 substitutions namely C60Y, N103K, G388K and W396R are lethal and GI specific of which former two are in domain II and the later two are in domain III. The lethality largely related to the overall destabilization of the protein, its malfunctioning and disease association.
These four substitutions affect most conserved region of the E protein. While in domain II, C60Y destroys a conserved di-sulfide bond, N103K occurs in the highly conserved fusion loop. G388K disrupts conserved RGD motif and W396R destroys most conserved tryptophan residue in domain III. N103K, the only lethal mutation which is common among all three GI/WB isolates (JEV28, JEV21 and JEV45) and earlier Ishikawa/JAPAN isolate, could be the reason for genotypic transition from existing GIII to newly emerge GI isolate in the region. Highly antigenic, top scored B-cell (both linear and conformational) and T-cell (both HLA class I and II) epitopes are seen to harbor these critical substitutions and thus these subset of epitopes may act as reference for design of GI specific vaccine. Although mutation pattern vary, these subset of epitopes act as target site for acquisition of genotypic transition related substitutions for other isolates of Asian continent. Overall our approaches highlight detailed aspects of immunoinformatics and find applications in the context of other infectious disease causing systems.

\section{Additional files}

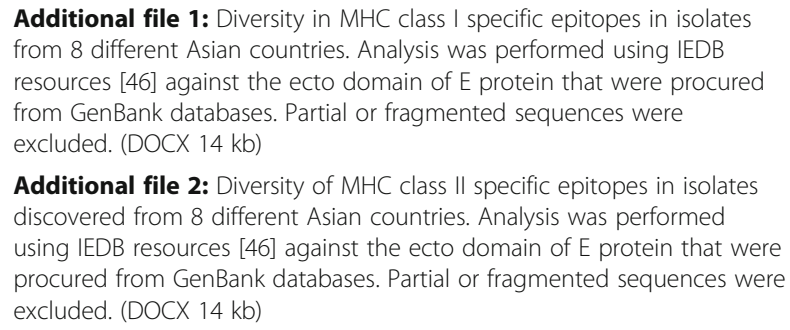

Additional file 1: Diversity in MHC class I specific epitopes in isolates from 8 different Asian countries. Analysis was performed using IEDB resources [46] against the ecto domain of E protein that were procured from GenBank databases. Partial or fragmented sequences were excluded. (DOCX $14 \mathrm{~kb}$ )

Additional file 2: Diversity of MHC class II specific epitopes in isolates discovered from 8 different Asian countries. Analysis was performed using IEDB resources [46] against the ecto domain of E protein that were procured from GenBank databases. Partial or fragmented sequences were excluded. (DOCX $14 \mathrm{~kb}$ )

\section{Abbreviations}

ACE: Atomic contact energy; AES: Acute encephalitis syndrome; ASA: Accessible surface area; C: Capsid C; CGE: Coarse-grained free-energy; CHARMM: Chemistry at HARvard macromolecular mechanics; DOPE: Discrete optimized protein energy; E protein: Envelope protein; EMBOSS: European molecular biology open software suite; GE: Global stabilization energy; Gl: Genotype I; GIII: Genotype III; GRAVY: Grand average hydropathy; HB: Hydrogen bonding; HLA: Human leukocyte antigen; HMM-SA: Hidden Markov Model-derived Structural Alphabet; JE: Japanese encephalitis; JEV: Japanese encephalitis virus; MHC: Major histocompatibility complex; MU: Mutant; NAMD: Nanoscale molecular dynamics; NT-MS site: N-terminal membrane attachment site; OPEP: Optimized potential for efficient protein structure prediction; PBG: Peptide-binding-groove; PDB: Protein data bank; PMDB: Protein model data base; prM: Precursor membrane; RMSD: Root mean square deviation; SDM: Site directed mutator; SNPs: Single nucleotide polymorphisms; TCR: T-cell receptor; Th: T-helper cell; UP: Uttar Pradesh; VDW: Van der waals; VMD: Visual molecular dynamics; WB: West Bengal; WT: Wild-type

\section{Acknowledgements}

Authors are grateful for the computer facility laboratory of DBT, Government of India in The Department of Biotechnology, The University of Burdwan. Authors are also thankful to Sudipta Mondal (Postgraduate student of The Department of Biotechnology, The University of Burdwan) for his help during bioinformatics analysis. 


\section{Funding}

No current funding sources for this study.

\section{Availability of data and materials}

The datasets supporting this study are included within the article and its additional files.

\section{Authors' contributions}

AKB conceived and designed the study. SB, PSSG and AKB wrote the manuscript. SB and PSSG performed work related to this manuscript. All authors read and approved the final manuscript.

\section{Competing interests}

The authors declare that they have no competing interests.

\section{Consent for publication}

Not applicable.

\section{Ethics approval and consent to participate}

Not applicable.

\section{Received: 18 July 2016 Accepted: 16 February 2017}

Published online: 06 March 2017

\section{References}

1. Saxena SK, Mishra N, Saxena R, Singh M, Mathur A. Trend of Japanese encephalitis in North India: evidence from thirty-eight acute encephalitis cases and appraisal of niceties. J Infect Dev Ctries. 2009;30:517-30.

2. Sarkar A, Banerjee S, Mukhopadhyay SK, Chatterjee S. Etiological spectrum of co-circulating Japanese encephalitis virus genotype I and III in clinically diagnosed AES cases from West Bengal, India: an indication of public health threat in near future. Int J Institutional Pharmacy Life Sci. 2015;5:154-180.

3. Sarkar A, Taraphdar D, Mukhopadhyay SK, Chakrabarti S, Chatterjee S. Molecular evidence for the occurrence of Japanese encephalitis virus genotype I and III infection associated with acute encephalitis in patients of West Bengal, India, 2010. Virol J. 2012;9:271.

4. Sarkar A, Banik A, Pathak BK, Mukhopadhyay SK, Chatterjee S. Envelope protein gene based molecular characterization of Japanese encephalitis virus clinical isolates from West Bengal, India: a comparative approach with respect to SA14-14-2 live attenuated vaccine strain. BMC Infect Dis. 2013;13: 368. doi:10.1186/1471-2334-13-368.

5. McMinn PC. The molecular basis of virulence of the encephalitogenic flaviviruses. J Gen Virol. 1997;78:2711-22

6. Mukhopadhyay S, Kuhn RJ, Rossmann MG. A structural perspective of the flavivirus life cycle. Nat Rev Microbiol. 2005;3:13-22. doi:10.1038/nrmicro1067.

7. Yu Y. Phenotypic and genotypic characteristics of Japanese encephalitis attenuated live vaccine virus SA14-14-2 and their stabilities. Vaccine. 2010; 28:3635-41. doi:10.1016/j.vaccine.2010.02.105.

8. Solomon T, Ni H, Beasley DW, Ekkelenkamp M, Cardosa MJ, Barrett AD Origin and evolution of Japanese encephalitis virus in Southeast Asia. J Virol. 2003;77:3091-8.

9. Fulmali PV, Sapkal GN, Athawale S, Gore MM, Mishra AC, Bondre VP. Introduction of Japanese encephalitis virus genotype I, India. Emerg Infect Dis. 2011;17:319-21.

10. Hu Q, Chen B, Zhu Z, Tian J, Zhou Y, Zhang X, et al. Recurrence of Japanese encephalitis epidemic in Wuhan, China, 2009-2010. PLoS One. 2013;8: e52687. doi:10.1371/journal.pone.0052687.

11. Zhang JS, Zhao QM, Guo XF, Zuo SQ, Cheng JX, Jia N, et al. Isolation and genetic characteristics of human genotype 1 Japanese encephalitis virus, China, 2009. PLoS One. 2011;6:e16418. doi:10.1371/journal.pone.0016418.

12. Cao QS, Li XM, Zhu QY, Wang DD, Chen HC, Qian P. Isolation and molecular characterization of genotype 1 Japanese encephalitis virus, SX09S-01, from pigs in China. Virol J. 2011;8:472. doi:10.1186/1743-422X-8-472

13. Nerome R, Tajima S, Takasaki T, Yoshida T, Kotaki A, Lim CK, et al. Molecular epidemiological analyses of Japanese encephalitis virus isolates from swine in Japan from 2002 to 2004. J Gen Virol. 2007:88:2762-8. doi:10.1099/vir.0.82941-0.

14. Monath TP, Arroyo J, Levenbook I, Zhang ZX, Catalan J, Draper K, et al. Single mutation in the flavivirus envelope protein hinge region increases neurovirulence for mice and monkeys but decreases viscerotropism for monkeys: relevance to development and safety testing of live, attenuated vaccines. J Virol. 2002;76:1932-43.
15. Ni H, Barrett AD. Molecular differences between wild-type Japanese encephalitis virus strains of high and low mouse neuroinvasiveness. J Gen Virol. 1996;77:1449-55. doi:10.1099/0022-1317-77-7-1449.

16. Rice P, Longden I, Bleasby A. EMBOSS: The European Molecular Biology Open Software Suite. Trends Genet. 2000;16:276-7.

17. Gupta PS, Banerjee S, Islam RN, Mondal S, Mondal B, Bandyopadhyay AK PHYSICO: An UNIX based Standalone Procedure for Computation of Individual and Group Properties of Protein Sequences. Bioinformation. 2013;10:105-7.

18. Banerjee S, Gupta PS, Nayek A, Das S, Sur VP, Seth P, et al. PHYSICO2: an UNIX based standalone procedure for computation of physicochemical, window-dependent and substitution based evolutionary properties of protein sequences along with automated block preparation tool, version 2. Bioinformation. 2015:11:366.

19. Geourjon C, Deleage G. SOPMA: significant improvements in protein secondary structure prediction by consensus prediction from multiple alignments. Comput Appl Biosci. 1995;11:681-4. doi:10.1093/bioinformatics/11.6.681.

20. Sen Gupta PS, Banerjee S, Bandyopadhyay AK. Sequence, structural and functional characterization of homogentisate-1, 2-dioxygenase of homo sapiens: an in silico analysis. Am J Bioinforma Res. 2013;3:42-61. doi:10.5923/.j.bioinformatics.20130303.02

21. Mondal S, Mondal B, Bandyopadhyay AK. Homology modeling of phaseolin from kidney bean (Phaseolus vulgaris L.): energy minimization and structure analysis. Int J Eng Sci Technol. 2013;5:992-8.

22. Sen Gupta PS, Banerjee S, Mondal S, Mondal B, Bandyopadhyay AK. An insight into the structure and function of chalcone synthase from sequence of Solanum tuberosum. J Adv Bioinforma Appl Res. 2014;5:8-22.

23. Sen Gupta PS, Mandal B, Bandyopadhyay AK. In silico characterization of human cyclooxygenase using computational tools and servers. Int J Institutional Pharmacy Life Sci. 2013:3:111.

24. Eswar N, Webb B, Marti-Renom MA, Madhusudhan MS, Eramian D, Shen MY et al. Comparative protein structure modeling using Modeller. Curr Protoc Bioinformatics. 2006. doi:10.1002/0471250953.bi0506s15. Chapter 5: Unit 5.6.

25. Shen MY, Andrej S. Statistical potential for assessment and prediction of protein structures. Protein Sci. 2006;15:2507-24. doi:10.1110/ps.062416606.

26. Phillips JC, Braun R, Wang W, Gumbart J, Tajkhorshid E, Villa E, et al. Scalable molecular dynamics with NAMD. J Comput Chem. 2005;26:1781-802. doi:10.1002/jcc.20289.

27. Humphrey W, Dalke A, Schulten K. VMD: visual molecular dynamics. J Mol Graph. 1996:14:33-8.

28. Pedretti A, Villa L, Vistoli G. VEGA: a versatile program to convert, handle and visualize molecular structure on Windows-based PCs. J Mol Graph Model. 2002;21:47-9. doi:10.1016/S1093-3263(02)00123-7.

29. Morris AL, MacArthur MW, Hutchinson EG, Thornton JM. Stereochemical quality of protein structure coordinates. Proteins: Struct, Funct, Bioinf. 1992; 12:345-64. doi:10.1002/prot.340120407.

30. Colovos C, Yeates TO. Verification of protein structures: patterns of nonbonded atomic interactions. Protein Sci. 1993;2:1511-9. doi:10.1002/pro.5560020916.

31. Bowie JU, Luthy $R$, Eisenberg D. A method to identify protein sequences that fold into a known three-dimensional structure. Science. 1991;253:164-70.

32. Wallner B, Elofsson A. Can correct protein models be identified? Protein Sci. 2003;12:1073-86. doi:10.1110/ps.0236803.

33. Gupta PS, Mondal S, Mondal B, Islam RN, Banerjee S, Bandyopadhyay AK SBION: A Program for Analyses of Salt-Bridges from Multiple Structure Files. Bioinformation. 2014;10:164-6. doi:10.6026/97320630010164.

34. Gupta PS, Nayek A, Banerjee S, Seth P, Das S, Sur VP, et al. SBION2: Analyses of Salt Bridges from Multiple Structure Files, Version 2. Bioinformation. 2015; 11:39-42. doi:10.6026/97320630011039.

35. Nayek A, Gupta PS, Banerjee S, Sur VP, Seth P, Das S, et al. ADSBET2: Automated Determination of Salt-Bridge Energy-Terms version 2. Bioinformation. 2015;11:413-5. doi:10.6026/97320630011413.

36. Castrignano T, De Meo PD, Cozzetto D, Talamo IG, Tramontano A. The PMDB protein model database. Nucleic Acids Res. 2006;34 Suppl 1:D306-9. doi:10.1093/nar/gkj105

37. Adzhubei IA, Schmidt S, Peshkin L, Ramensky VE, Gerasimova A, Bork P, et al. A method and server for predicting damaging missense mutations. Nat Methods. 2010;7:248-9. doi:10.1038/nmeth0410-248.

38. Capriotti E, Fariselli P, Calabrese R, Casadio R. Predicting protein stability changes from sequences using support vector machines. Bioinformatics. 2005:21 Suppl 2:ii54-58.

39. Johnson AD, Handsaker RE, Pulit SL, Nizzari MM, O'Donnell CJ, de Bakker PI. SNAP: a web-based tool for identification and annotation of proxy SNPs using HapMap. Bioinformatics. 2008;24:2938-9. doi:10.1093/bioinformatics/btn564. 
40. Capriotti E, Altman RB, Bromberg Y. Collective judgment predicts diseaseassociated single nucleotide variants. BMC Genomics. 2013;14 Suppl 3:S2. doi:10.1186/1471-2164-14-S3-S2.

41. Topham CM, Srinivasan N, Blundell TL. Prediction of the stability of protein mutants based on structural environment-dependent amino acid substitution and propensity tables. Protein Eng. 1997;10:7-21. doi:10.1093/protein/10.1.7.

42. Larsen JE, Lund O, Nielsen M. Improved method for predicting linear B-cell epitopes. Immunome Res. 2006;2:2. doi:10.1186/1745-7580-2-2.

43. Thevenet P, Shen Y, Maupetit J, Guyon F, Derreumaux P, Tuffery P. PEPFOLD: an updated de novo structure prediction server for both linear and disulfide bonded cyclic peptides. Nucleic Acids Res. 2012;40(Web Server issue):W288-93. doi:10.1093/nar/gks419.

44. Hubbard SJ, Thornton JM. NACCESS, Computer Program. 1993. Department of Biochemistry and Molecular Biology, University College London.

45. Liang S, Zheng D, Standley DM, Yao B, Zacharias M, Zhang C. EPSVR and EPMeta: prediction of antigenic epitopes using support vector regression and multiple server results. BMC Bioinf. 2010;11:381. doi:10.1186/1471-2105-11-381.

46. Zhang Q, Wang P, Kim Y, Haste-Andersen P, Beaver J, Bourne PE, et al. Immune epitope database analysis resource (IEDB-AR). Nucleic Acids Res. 2008;36 Suppl 2:W513-8. doi:10.1093/nar/gkn254.

47. Doytchinova IA, Flower DR. VaxiJen: a server for prediction of protective antigens, tumour antigens and subunit vaccines. BMC Bioinf. 2007:8:4. doi:10.1186/1471-2105-8-4

48. Camproux AC, Tuffery P. Hidden Markov model-derived structural alphabet for proteins: the learning of protein local shapes captures sequence specificity. Biochim Biophys Acta. 2005;1724:394-403. doi:10.1016/j.bbagen.2005.05.019.

49. Sahu TK, Rao AR, Meher PK, Sahoo BC, Gupta S, Rai A. Computational prediction of $\mathrm{MHC}$ class I epitopes for most common viral diseases in cattle (Bos taurus). Indian J Biochem Biophys. 2015;52:34-44.

50. Schneidman-Duhovny D, Inbar Y, Nussinov R, Wolfson HJ. PatchDock and SymmDock: servers for rigid and symmetric docking. Nucleic Acids Res. 2005;33(Web Server issue):W363-7. doi:10.1093/nar/gki481.

51. Mashiach E, Schneidman-Duhovny D, Andrusier N, Nussinov R, Wolfson HJ. FireDock: Fast Interaction Refinement in Molecular Docking. Nucleic Acids Res. 2008;36(Web Server issue):W229-32. doi:10.1093/nar/gkn186.

52. Luca VC, AbiMansour J, Nelson CA, Fremont DH. Crystal structure of the Japanese encephalitis virus envelope protein. J Virol. 2012;86:2337-46. doi:10.1128/JVI.06072-11.

53. Wu KP, Wu CW, Tsao YP, Kuo TW, Lou YC, Lin CW, et al. Structural basis of a flavivirus recognized by its neutralizing antibody: solution structure of the domain III of the Japanese encephalitis virus envelope protein. J Biol Chem. 2003;278:46007-13. doi:10.1074/jbc.M307776200.

54. Baker D, Sali A. Protein structure prediction and structural genomics. Science. 2001;294:93-6.

55. Zhang C, Kim SH. Overview of structural genomics: from structure to function. Curr Opin Chem Biol. 2003;7:28-32.

56. Murzin AG, Patthy L. Sequences and topology From sequence to structure to function. Curr Opin Struct Biol. 1999;9:359-62. doi:10.1016/S0959-440X(99)80048.

57. Sun P, Ju H, Liu Z, Ning Q, Zhang J, Zhao X, et al. Bioinformatics resources and tools for conformational B-cell epitope prediction. Comput Math Methods Med. 2013;2013:943636. doi:10.1155/2013/943636.

58. Worth CL, Burke DF, Blundell TL. Estimating the effects of single nucleotide polymorphisms on protein structure: how good are we at identifying likely disease associated mutations? Proceedings of Molecular Interactions Bringing Chemistry to Life. 2007. p. 11-26.

59. Sultana H, Foellmer HG, Neelakanta G, Oliphant T, Engle M, Ledizet $M$, et al. Fusion loop peptide of the West Nile virus envelope protein is essential for pathogenesis and recognized by a therapeutic cross-reactive human monoclonal antibody. J Immunol. 2009;183:650-60. doi:10.4049/jimmunol.0900093.

60. Lobigs M, Usha R, Nestorowicz A, Marshall ID, Weir RC, Dalgarno L. Host cell selection of Murray Valley encephalitis virus variants altered at an RGD sequence in the envelope protein and in mouse virulence. Virology. 1990; 176:587-95. doi:10.1016/0042-6822(90)90029-Q.

61. Melo F, Devos D, Depiereux E, Feytmans E. ANOLEA: a www server to assess protein structures. Proc Int Conf Intell Syst Mol Biol. 1997:5:187-90.

62. Vaughan K, Greenbaum J, Blythe M, Peters B, Sette A. Meta-analysis of all immune epitope data in the Flavivirus genus: inventory of current immune epitope data status in the context of virus immunity and immunopathology. Viral Immunol. 2010;23:259-84. doi:10.1089/vim.2010.0006.
63. Cecilia D, Gadkari DA, Kedarnath N, Ghosh SN. Epitope mapping of Japanese encephalitis virus envelope protein using monoclonal antibodies against an Indian strain. J Gen Virol. 1988;69:2741-7. doi:10.1099/0022-1317-69-11-2741.

64. Wang F, Feng X, Zheng Q, Hou H, Cao R, Zhou B, et al. Multiple linear epitopes (B-cell, CTL and Th) of JEV expressed in recombinant MVA as multiple epitope vaccine induces a protective immune response. Virol J. 2012;9:204. doi:10.1186/1743-422X-9-204.

65. Yao B, Zheng D, Liang S, Zhang C. Conformational B-cell epitope prediction on antigen protein structures: a review of current algorithms and comparison with common binding site prediction methods. PLoS One. 2013;8:e62249. doi:10.1371/journal.pone.0062249.

66. De Filette M, Chabierski S, Andries O, Ulbert S, Sanders NN. T cell epitope mapping of the e-protein of West Nile virus in BALB/c mice. PLoS One. 2014;9:e115343. doi:10.1371/journal.pone.0115343.

67. Takada K, Masaki H, Konishi E, Takahashi M, Kurane I. Definition of an epitope on Japanese encephalitis virus (JEV) envelope protein recognized by JEVspecific murine CD8+ cytotoxic T lymphocytes. Arch Virol. 2000;145:523-34.

68. Quinan BR, Flesch IE, Pinho TM, Coelho FM, Tscharke DC, da Fonseca FG. An intact signal peptide on dengue virus $E$ protein enhances immunogenicity for CD8(+) T cells and antibody when expressed from modified vaccinia Ankara. Vaccine. 2014;32:2972-90. doi:10.1016/j.vaccine.2014.03.093.

69. Rivino L, Kumaran EA, Jovanovic V, Nadua K, Teo EW, Pang SW, et al. Differential targeting of viral components by CD4+ versus CD8+ T lymphocytes in dengue virus infection. J Virol. 2013;87:2693-706. doi:10.1128/JVI.02675-12.

70. Sidney J, Grey HM, Southwood S, Celis E, Wentworth PA, del Guercio MF, et al. Definition of an HLA-A3-like supermotif demonstrates the overlapping peptide-binding repertoires of common HLA molecules. Hum Immunol. 1996;45:79-93. doi:10.1016/0198-8859(95)00173-5.

71. Nayek A, Sen Gupta PS, Banerjee S, Mondal B, Bandyopadhyay AK. Salt-Bridge Energetics in halophilic Proteins. Plos One. 2014;9:e93862. doi:10.1371/journal.pone.0093862.

72. Gromowski GD, Firestone CY, Whitehead SS. Genetic Determinants of Japanese Encephalitis Virus Vaccine Strain SA14-14-2 That Govern Attenuation of Virulence in Mice. J Virol. 2015;89:6328-37. doi:10.1128/JVI.00219-15.

73. Nitayaphan S, Grant JA, Chang GJ, Trent DW. Nucleotide sequence of the virulent SA-14 strain of Japanese encephalitis virus and its attenuated vaccine derivative, SA14-14-2. Virology. 1990;177:541-52. doi:10.1016/0042-6822(90)90519-W.

74. Betts MJ, Russell RB. Chapter 14. Amino acid properties and consequences of substitutions. In: Barnes MR, Gray IC, editors. Bioinformatics for Geneticists. Wiley; 2003. doi:10.1002/0470867302.ch14.

75. Lin HE, Tsai WY, Liu IJ, Li PC, Liao MY, Tsai JJ, et al. Analysis of epitopes on dengue virus envelope protein recognized by monoclonal antibodies and polyclonal human sera by a high throughput assay. PLoS Negl Trop Dis. 2012;6:e1447. doi:10.1371/journal.pntd.0001447.

76. Darwin C. On the origin of species by means of natural selection: Or the preservation of favoured races in the struggle for life (Special ed.). Birmingham: Gryphon Editions; 1987; 1859.

77. Duret L. Neutral theory: The null hypothesis of molecular evolution. Nature Education. 2008;1:803-6. 218.

78. Alberts B, Johnson A, Lewis J, Raff M, Roberts K, Walter P. Molecular Biology of the Cell. 4th ed. New York: Garland Science; 2002.

79. Mercado ES, Espino FE, Perez MLM, Bilar JM, Bajaro JDP, Huy NT, et al. HLAA33:01 as Protective Allele for Severe Dengue in a Population of Filipino Children. PLoS One. 2015;10:e0115619. doi:10.1371/journal.pone.0115619.

80. Thanapati S, Hande A, Das R, Gurav Y, Tripathy AS. Association of human leukocyte antigen class II allele and haplotypes in chikungunya viral infection in a western Indian population. Trans R Soc Trop Med Hyg. 2014; 108:277-82. doi:10.1093/trstmh/tru030.

81. Doganay L, Fejzullahu A, Katrinli S, Yilmaz Enc F, Ozturk O, Colak Y, Ulasoglu C, et al. Association of human leukocyte antigen DQB1 and DRB1 alleles with chronic hepatitis B. World J Gastroenterol. 2014;20: 8179-86. doi:10.3748/wjg.v20.i25.8179. 